\title{
BIFINITE CHU SPACES
}

\author{
MANFRED DROSTE AND GUO-QIANG ZHANG
}

Institute of Computer Science, Leipzig University, 04158 Leipzig, Germany

e-mail address: droste@informatik.uni-leipzig.de

Department of Electrical Engineering and Computer Science, Case Western Reserve University, Cleveland, $\mathrm{OH}$ 44106, U.S.A.

e-mail address: gq@case.edu

\begin{abstract}
This paper studies colimits of sequences of finite Chu spaces and their ramifications. Besides generic Chu spaces, we consider extensional and biextensional variants. In the corresponding categories we first characterize the monics and then the existence (or the lack thereof) of the desired colimits. In each case, we provide a characterization of the finite objects in terms of monomorphisms/injections. Bifinite Chu spaces are then expressed with respect to the monics of generic Chu spaces, and universal, homogeneous Chu spaces are shown to exist in this category. Unanticipated results driving this development include the fact that while for generic Chu spaces monics consist of an injective first and a surjective second component, in the extensional and biextensional cases the surjectivity requirement can be dropped. Furthermore, the desired colimits are only guaranteed to exist in the extensional case. Finally, not all finite Chu spaces (considered set-theoretically) are finite objects in their categories. This study opens up opportunities for further investigations into recursively defined Chu spaces, as well as constructive models of linear logic.
\end{abstract}

\section{INTRODUCTION}

Within semantic frameworks for programming languages, a basic approach to the study of infinite objects is through their finite approximations. This is true both within an individual domain, as well as with domains collectively. A salient example of the latter is Plotkin's approach to SFP [15], where a class of domains is constructed systematically by taking colimits of sequences of finite partial orders. An important component of this framework is the notion of embedding-projection pair, capturing when one partial order is an approximation of another. An interesting outcome of this process is that completeness of an individual domain, the property that makes a cpo complete, becomes a natural by-product obtained by taking colimits of finite structures. In domain theory, the SFP- (or bifinite) domains now form an important cartesian closed category of domains, see [1].

In this paper we study colimits of sequences of finite Chu spaces. This entails the use of monic morphisms (or monomorphisms) as a way to formulate the substructure relationship. Such an innocuous attempt led to striking differentiations of the notion dictated by the

1998 ACM Subject Classification: F.3.2.

2000 Mathematics Subject Classification: 03B70, 06A15, 06B23, 08A70, 68P99, 68Q55.

Key words and phrases: Domain theory, approximation, category theory, Chu spaces. 
extensionality properties of the underlying spaces. We consider three base categories of Chu spaces: the generic Chu spaces $(\mathbf{C})$, the extensional Chu spaces $(\mathbf{E})$, and the biextensional Chu spaces (B). The main results are: (1) a characterization of monics in each of the three categories; (2) existence (or the lack thereof) of colimits and a characterization of finite objects in each of the corresponding categories using monomorphisms/injections (denoted as $\mathbf{i C}, \mathbf{i E}$, and $\mathbf{i B}$, respectively); (3) a formulation of bifinite Chu spaces with respect to iC; (4) the existence of universal, homogeneous Chu spaces in this category. Unanticipated results driving this development include the fact that: (a) in $\mathbf{C}$, a morphism $(f, g)$ is monic iff $f$ is injective and $g$ is surjective while for $\mathbf{E}$ and $\mathbf{B},(f, g)$ is monic iff $f$ is injective (but $g$ is not necessarily surjective); (b) while colimits always exist in $\mathbf{i E}$, it is not the case for iC and iB; (c) not all finite Chu spaces (considered set-theoretically) are finite objects in their categories.

Bifinite Chu spaces can be viewed, in an intuitive category-theoretic sense, as "countable" objects which are approximable by the finite objects of the category. The class of bifinite Chu spaces is very rich (up to isomorphism, there are uncountably many such spaces). However, we show that there is a single bifinite Chu space $U$ which contains any other bifinite Chu space as a subspace. Moreover, $U$ can be chosen to be homogeneous, i.e. to bear maximal possible degree of symmetry, and with this additional property $U$ is unique up to isomorphism.

Our interest in Chu spaces stems from a number of recent developments. Chu spaces provide a suitable model of linear logic, originating from a general categorical construction introduced by Barr and his student [2, 3]. The rich mathematical content of Chu spaces has been extensively illustrated by Pratt and his collaborators in a variety of settings, ranging from concurrency to logic and category theory [18, 19, 20, 21, 23, 24]. In particular, Pratt shows that all small categories can be embedded in a certain category of Chu spaces [22].

Chu spaces are closely related to the topic of Formal Concept Analysis (FCA [8, 27]). Both areas use the same objects but the morphisms considered in FCA are different. Chu spaces are also related to domains [27]. In [13], a class called casuistries was introduced as a "continuous" version of Chu spaces, and yet maintaining the constructions desired as a model of linear logic. On the other hand, if instead of Chu transformations, Chu spaces are equipped with what are called approximable mappings [9, 26], one obtains a cartesian closed category equivalent to the category of algebraic lattices and Scott continuous functions [10]. For this to work properly, a modified notion of formal concept, called approximable concept, needs to be used [28]. This way, an infinite concept can be approximated by finite ones.

Universal objects have played an important role in the development of domain theory. For example, the early work of Scott [25] and Plotkin [16] showed that with universal objects, domain equations can be treated by a calculus of retracts.

By studying Chu spaces that are colimits of sequences of finite objects, we hope to understand these spaces from a constructive angle, formulate a notion of completeness, and study the existence of universal, homogeneous objects. Recursively defined Chu spaces as well as models of linear logic within bifinite Chu spaces are some topics worth revisiting in light of this paper.

The rest of the paper is organized as follows. Section 2 recalls basic terminologies and gives a characterization of monic morphisms in the categories $\mathbf{C}, \mathbf{E}$, and $\mathbf{B}$. Section 3 studies colimits in the categories $\mathbf{i C}, \mathbf{i E}$, and $\mathbf{i B}$. Section 4 characterizes finite objects in $\mathbf{i C}$, $\mathbf{i E}$, and iB. Section 5 introduces bifinite Chu spaces and shows the existence of universal, homogeneous bifinite Chu spaces using finite amalgamation. 
Remark: The shortened conference version of the paper was presented at CALCO 2007 in Bergen, Norway.

\section{Chu SPACES AND MONiC MORPhisms}

We recall some basic definitions to fix notation, following [4]. Readers interested in more details should consult [19]. In [19], the morphisms are called Chu transformations.

Definition 2.1. A Chu space over a set $\Sigma$ is a triple $(A, r, X)$ where $A$ is a set whose elements can be considered as objects and $X$ is a set whose elements can be regarded as attributes. The satisfaction relation $r$ is a function $A \times X \rightarrow \Sigma$. A morphism from a Chu space $(A, r, X)$ to a Chu space $(B, s, Y)$ is a pair of functions $(f, g)$, with $f: A \rightarrow B$ and $g: Y \rightarrow X$ such that for any $a \in A$ and $y \in Y, s(f(a), y)=r(a, g(y))$. To alleviate the notational burden, we refer to a morphism by $\varphi=(f, g)$, and refer to the forward component by $\varphi^{+}=f$ and the backward component by $\varphi^{-}=g$.

For all the examples we consider in this paper, $\Sigma=\{0,1\}$. If $\Sigma$ is left unspecified, then it is assumed to contain at least two elements, denoted as 0 and 1 . A Chu space $(A, r, X)$ has two equivalence relations built-in. One is on the rows, where the $a$-th row corresponds to a function $r(a,-): X \rightarrow \Sigma$. Two rows $a, b$ are equivalent if $r(a,-)=r(b,-)$. Similarly, an equivalence relation exists on columns, defined by equality $r(-, x)=r(-, y)$ for $x, y \in X$. A Chu space $(A, r, X)$ is called extensional if $r(-, x)=r(-, y)$ implies $x=y$, i.e., $r$ does not contain repeated columns. Similarly, a Chu space $(A, r, X)$ is separable if it does not contain repeated rows. Using topological analogy, if we think of objects in $A$ as points and attributes in $X$ as open sets, then separable Chu spaces are those for which distinct points can be differentiated by the open sets containing them (such spaces are called $T_{0}$ ). A Chu space is biextensional if it is both separable and extensional.

We denote by $\mathbf{C}$ the category of $\mathbf{C h u}$ spaces and morphisms defined above, and $\mathbf{E}$ and $\mathbf{B}$ the full subcategories of extensional and biextensional Chu spaces, respectively. Composition of morphisms reduces to functional compositions of the components: $\varphi_{2} \circ \varphi_{1}=$ $\left(\varphi_{2}^{+} \circ \varphi_{1}^{+}, \varphi_{1}^{-} \circ \varphi_{2}^{-}\right)$, noting that the second component goes backwards. For abbreviation, objects are denoted as $\mathrm{C}_{i}$ for short, where $\mathrm{C}_{i}:=\left(A_{i}, r_{i}, X_{i}\right)$. We refer to $A_{i}$ the object set, and $X_{i}$ as the attribute set of $C_{i}$, respectively. As a refinement of an observation in [13, we have the following result which will be useful for subsequent developments of the paper.

Proposition 2.1. Suppose $\varphi_{1}, \varphi_{2}: \mathrm{C} \rightarrow \mathrm{C}^{\prime}$ are morphisms in $\mathbf{C}$. Then

(1) if $\mathrm{C}$ is extensional, then $\varphi_{1}^{+}=\varphi_{2}^{+}$implies $\varphi_{1}^{-}=\varphi_{2}^{-}$;

(2) if $\mathrm{C}^{\prime}$ is separable, then $\varphi_{1}^{-}=\varphi_{2}^{-}$implies $\varphi_{1}^{+}=\varphi_{2}^{+}$;

(3) if $\mathrm{C}$ and $\mathrm{C}^{\prime}$ are biextensional, then $\varphi_{1}^{-}=\varphi_{2}^{-}$iff $\varphi_{1}^{+}=\varphi_{2}^{+}$.

Thus, the forward and backward components in a morphism determine each other uniquely in the category of biextensional Chu spaces.

Proof. Let us write $\mathbf{C}=(A, r, X)$ and $C^{\prime}=\left(A^{\prime}, r^{\prime}, X^{\prime}\right)$. First we show (1). Suppose $\mathbf{C}$ is extensional and $\varphi_{1}^{+}=\varphi_{2}^{+}$. Then for all $x^{\prime} \in X^{\prime}$ and $a \in A$ we have

$$
r\left(a, \varphi_{1}^{-}\left(x^{\prime}\right)\right)=r^{\prime}\left(\varphi_{1}^{+}(a), x^{\prime}\right)=r^{\prime}\left(\varphi_{2}^{+}(a), x^{\prime}\right)=r\left(a, \varphi_{2}^{-}\left(x^{\prime}\right)\right)
$$

Hence $\varphi_{1}^{-}\left(x^{\prime}\right)=\varphi_{2}^{-}\left(x^{\prime}\right)$ by extensionality of C. Now (2) follows from (1) by duality, and (1) and (2) imply (3). 
As a first order of business, we consider monic morphisms, which capture the notion of a "substructure". In categorical terms, a morphism $\varphi: C_{1} \rightarrow C_{2}$ is monic (or mono) if for any other morphisms $\varphi_{i}: \mathrm{C}^{*} \rightarrow \mathrm{C}_{1}(i=1,2)$ such that $\varphi \circ \varphi_{1}=\varphi \circ \varphi_{2}$, we have $\varphi_{1}=\varphi_{2}$.

Remark. To make a distinction in our reference to morphisms at different levels, we reserve the term monic, mono, epi, etc for Chu spaces, and use one-to-one, onto, injective, surjective for the functions on the underlying sets. When properties on the underlying functions carry over to Chu spaces, we occasionally mix the terms.

Proposition 2.2. We have:

(1) A morphism $\varphi: \mathbf{C} \rightarrow \mathrm{C}^{\prime}$ in $\mathbf{C}$ is monic iff $\varphi^{+}$is injective and $\varphi^{-}$is surjective.

(2) A morphism $\varphi: \mathbf{C} \rightarrow \mathrm{C}^{\prime}$ in $\mathbf{E}$ is monic iff $\varphi^{+}$is injective.

(3) A morphism $\varphi: \mathbf{C} \rightarrow \mathbf{C}^{\prime}$ in $\mathbf{B}$ is monic iff $\varphi^{+}$is injective.

(4) Suppose $\varphi:(A, r, X) \rightarrow(B, s, Y)$ is a morphism in $\mathbf{C}$ and $(B, s, Y)$ is extensional. If $\varphi^{+}$is surjective, then $\varphi^{-}$is injective.

Proof. (1) The "If" part is straightforward. We check the "Only If" part. Suppose $\varphi: C \rightarrow$ $\mathrm{C}^{\prime}$ is such that for any pair of morphisms $\varphi_{i}: \mathrm{C}^{*} \rightarrow \mathrm{C}, i=1,2$, if $\varphi \circ \varphi_{1}=\varphi \circ \varphi_{2}$, then $\varphi_{1}=\varphi_{2}$. We show that $\varphi^{+}$is injective and $\varphi^{-}$is surjective. Let's write $C=(A, r, X)$ and $\mathrm{C}^{\prime}=\left(A^{\prime}, r^{\prime}, X^{\prime}\right)$.

First we show that $\varphi^{-}$is surjective. Suppose otherwise. Choose two sets $X_{1}, X_{2}$ of the same cardinality as $X \backslash \varphi^{-}\left(X^{\prime}\right)$ such that $\varphi^{-}\left(X^{\prime}\right), X_{1}, X_{2}$ are pairwise disjoint. Put $X^{*}=\varphi^{-}\left(X^{\prime}\right) \cup X_{1} \cup X_{2}$ and $A^{*}=A$. For $i=1,2$, choose a bijection $g_{i}: X \backslash \varphi^{-}\left(X^{\prime}\right) \rightarrow X_{i}$ and let $g_{i}^{*}=i d_{\varphi^{-}\left(X^{\prime}\right)} \cup g_{i}: X \rightarrow X^{*}$ be the joint extension of the identity $i d_{\varphi^{-}\left(X^{\prime}\right)}$ on $\varphi^{-}\left(X^{\prime}\right)$ and $g_{i}$. Let $f^{*}=i d_{A}$. For $a \in A^{*}$ and $x \in X^{*}$, let $r^{*}(a, x)=r(a, x)$ if $x \in \varphi^{-}\left(X^{\prime}\right)$, $r^{*}(a, x)=r\left(a, g_{1}^{-1}(x)\right)$ if $x \in X_{1}$, and $r^{*}(a, x)=r\left(a, g_{2}^{-1}(x)\right)$ if $x \in X_{2}$.

Let $\mathrm{C}^{*}=\left(A^{*}, r^{*}, X^{*}\right)$. Then $\left(f^{*}, g_{i}^{*}\right): \mathrm{C}^{*} \rightarrow \mathrm{C}$, with $i=1,2$, are morphisms. Indeed, $r\left(f^{*}(a), x\right)=r(a, x)=r^{*}\left(a, g_{i}^{*}(x)\right)$ if $x \in \varphi^{-}\left(X^{\prime}\right)$, and $r\left(f^{*}(a), x\right)=r(a, x)=r^{*}\left(a, g_{i}(x)\right)$ also, if $x \in X \backslash \varphi^{-}\left(X^{\prime}\right)$, for $i=1,2$ by the definition of $r^{*}$. Moreover, $\left(f^{*}, g_{i}^{*}\right)$ yield the same composition with $\varphi$ because $g_{i}^{*}$ s behave the same on the image set $\varphi^{-}\left(X^{\prime}\right)$. Now $\varphi$ being monic implies that $g_{1}=g_{2}$, a contradiction because $X \backslash \varphi^{-}\left(X^{\prime}\right) \neq \emptyset$. Hence $\varphi^{-}\left(X^{\prime}\right)=X$. Note that for this construction to work, $C^{*}$ cannot be required to be extensional.

The proof for the injectivity of $\varphi^{+}$is the same as the "only if" part for item (2), given next.

(2) and (3). If. Let $\varphi: C \rightarrow C^{\prime}$ be a morphism and $\varphi^{+}$an injection. Consider two morphisms $\varphi_{i}: \mathrm{C}^{*} \rightarrow \mathrm{C}(i=1,2)$, which yield the same compositions with $\varphi$. Then $\varphi_{1}^{+}=\varphi_{2}^{+}$, since $\varphi^{+}$is injective. By Prop. 2.1, we have $\varphi_{1}^{-}=\varphi_{2}^{-}$. Hence $\varphi$ is monic.

Only If. Let $\varphi: \mathrm{C} \rightarrow \mathrm{C}^{\prime}$ be monic and write $\mathrm{C}=(A, r, X)$ and $\mathrm{C}^{\prime}=\left(A^{\prime}, r^{\prime}, X^{\prime}\right)$. Assume $a_{1}, a_{2} \in A$ are such that $\varphi^{+}\left(a_{1}\right)=\varphi^{+}\left(a_{2}\right)=a^{\prime}$. Construct a Chu space $\mathrm{C}^{*}=\left(A^{*}, r^{*}, X^{*}\right)$ as follows. Let $A^{*}=\{a\}$, a singleton, and $X^{*}=\Sigma$. Also, let $r^{*}(a, \sigma)=\sigma$ for $\sigma \in \Sigma$. Clearly, $\mathrm{C}^{*}$ is biextensional.

Now define $\varphi_{i}: \mathrm{C}^{*} \rightarrow \mathrm{C}, i=1,2$, as follows. Let $\varphi_{i}^{+}(a)=a_{i}$ for $i=1,2$. For $x \in X$, put $\varphi_{i}^{-}(x)=r\left(a_{i}, x\right) \in \Sigma=X^{*}(i=1,2)$. Then $\varphi_{1}, \varphi_{2}: \mathrm{C}^{*} \rightarrow \mathrm{C}$ are morphisms. Clearly, $\varphi^{+} \circ \varphi_{1}^{+}=\varphi^{+} \circ \varphi_{2}^{+}$. We claim that also $\varphi_{1}^{-} \circ \varphi^{-}=\varphi_{2}^{-} \circ \varphi^{-}$. Indeed, if $x^{\prime} \in X^{\prime}$ and $x=\varphi^{-}\left(x^{\prime}\right)$, then

$$
\varphi_{1}^{-}(x)=r\left(a_{1}, x\right)=r^{\prime}\left(a^{\prime}, x^{\prime}\right)=r\left(a_{2}, x\right)=\varphi_{2}^{-}(x) .
$$

Hence $\varphi_{1}, \varphi_{2}: \mathrm{C}^{*} \rightarrow \mathrm{C}$ yield the same compositions with $\varphi$. Since $\varphi$ is monic, it follows that $\varphi_{1}^{+}=\varphi_{2}^{+}$. Thus $a_{1}=a_{2}$, and $\varphi^{+}$is injective. 
(4) Let $y, y^{\prime} \in Y$ with $\varphi^{-}(y)=\varphi^{-}\left(y^{\prime}\right)=x$, say. Choose any $b \in B$ and then $a \in A$ with $\varphi^{+}(a)=b$. Then $s(b, y)=r(a, x)=s\left(b, y^{\prime}\right)$. Then $y=y^{\prime}$ by extensionality.

The second and third items above would not be so surprising if the injectivity of $\varphi^{+}$ implied the surjectivity of $\varphi^{-}$in $\mathbf{B}$ and $\mathbf{E}$. But this is not the case.

Example 2.3. Consider $\mathrm{C}:=\left(\{a\}, r,\left\{x_{1}, x_{2}\right\}\right)$, with $r\left(a, x_{1}\right)=0$ and $r\left(a, x_{2}\right)=1 ; \mathrm{C}^{\prime}:=$ $\left(\{b\}, r^{\prime},\{y\}\right)$, with $r^{\prime}(b, y)=0$. Then the constraints $f(a)=b$ and $g(y)=x_{1}$ satisfy the property that $r^{\prime}(f(a), y)=0=r(a, g(y))$ and the pair $(f, g)$ gives rise to a morphism. Clearly $\mathbf{C}, \mathbf{C}^{\prime} \in \mathbf{B}$ and $f$ is injective, but $g$ is not surjective. With respect to items (2) and (3) in the proposition, this means that the backward component of a monic morphism in $\mathbf{E}$ and $\mathbf{B}$ need not be surjective.

Remark. Using a similar proof, we can show that a morphism $\varphi: C \rightarrow C^{\prime}$ is monic if and only if $\varphi^{-}$is surjective, in the category of separable Chu spaces. We omitted this statement in Prop. 2.2 because we do not consider the category of separable Chu spaces in the rest of the paper.

\section{Colimits of $\omega$-Chains}

We are interested in the subcategories of $\mathbf{C}, \mathbf{E}$, and $\mathbf{B}$ with monic morphisms, denoted as $\mathbf{i C}, \mathbf{i E}$, and $\mathbf{i B}$, respectively. Let us begin with an unexpected observation that colimits do not exist in $\mathbf{i C}$ in general. For this purpose, we recall the definition of colimits, here formulated in $\mathbf{i C}$, but it can easily be seen as an instantiation of a general notion [14. We then show that colimits do exist in $\mathbf{i E}$ and $\mathbf{i B}$.

Definition 3.1. An $\omega$-sequence in $\mathbf{i C}$ is a family $\left(\mathrm{C}_{i}, \varphi_{i}\right)_{i \geq 1}$

$$
\mathrm{C}_{1} \stackrel{\varphi_{1}}{\longrightarrow} \mathrm{C}_{2} \stackrel{\varphi_{2}}{\longrightarrow} \mathrm{C}_{3} \stackrel{\varphi_{3}}{\longrightarrow} \cdots \mathrm{C}_{i-1} \stackrel{\varphi_{i-1}}{\longrightarrow} \mathrm{C}_{i} \stackrel{\varphi_{i}}{\longrightarrow} \mathrm{C}_{i+1} \cdots
$$

Definition 3.2. A cocone from an $\omega$-sequence $\left(C_{i}, \varphi_{i}\right)_{i \geq 1}$ to a Chu space $\mathrm{C}:=(A, r, X)$ is a family of mappings $\mathrm{C}_{i} \stackrel{\psi_{i}}{\longrightarrow} \mathrm{C}$ such that $\psi_{i+1} \circ \varphi_{i}=\psi_{i}$, for all $i \geq 1$, i.e., the diagram

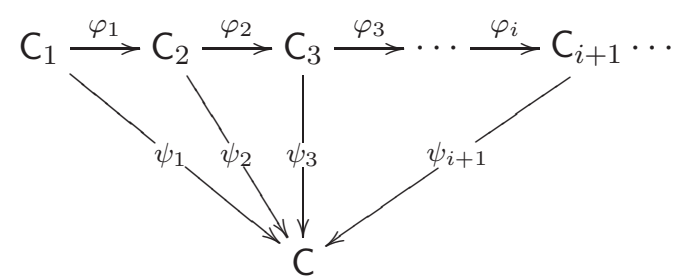

commutes.

A cocone $\left(\mathrm{C}_{i} \stackrel{\psi_{i}}{\longrightarrow} \mathrm{C}\right)_{i \geq 1}$ is universal if for any other cocone $\left(\mathrm{C}_{i} \stackrel{\psi^{\prime} i}{\longrightarrow} \mathrm{C}^{\prime}\right)_{i \geq 1}$ such that $\psi_{i+1}^{\prime} \circ \varphi_{i}=\psi_{i}^{\prime}$ for all $i \geq 1$, there exists a unique $\mathrm{C} \stackrel{\psi}{\longrightarrow} \mathrm{C}^{\prime}$ such that $\psi \circ \psi_{i}=\psi_{i}^{\prime}$ for all $i \geq 1$. Such a universal cocone, if it exists, is called the colimit of the family $\left(C_{i}, \varphi_{i}\right)_{i \geq 1}$, while $\psi$ is called the mediating map. In this case we write $C=\operatorname{colim}_{i}\left(C_{i}, \varphi_{i}\right)$.

Theorem 3.1. Colimits of $\omega$-chains of finite Chu spaces do not always exist in iC.

Proof. Consider finite Chu spaces $\mathrm{C}_{i}:=\left(\{1, \ldots, i\}, r_{i},\{1, \ldots, i\}\right)$, such that $r_{i}(a, x)=1$ if $a \leq x$, and $r_{i}(a, x)=0$ otherwise. Observe that $C_{i}$ is biextensional. Define $\varphi_{i}: \mathrm{C}_{i} \rightarrow \mathrm{C}_{i+1}$ such that $\varphi_{i}^{+}(a)=a$ for $a=1, \ldots, i$, and $\varphi_{i}^{-}(i+1)=i$, but $\varphi_{i}^{-}(x)=x$ otherwise. It is straightforward to verify that the $\varphi_{i}$ s are indeed morphisms: for all $1 \leq a \leq i$ and 
$1 \leq x \leq i+1, r_{i+1}\left(\varphi_{i}^{+}(a), x\right)=1$ iff $r_{i+1}(a, x)=1$ iff $a \leq x$ iff $r_{i}\left(a, \varphi_{i}^{-}(x)\right)=1$. Hence $\varphi_{i}$ is monic.

Consider $\mathrm{C}:=(\mathbb{N}, r, \mathbb{N})$, with $r(a, x)=1$ iff $a \leq x$, and $r(a, x)=0$ otherwise. Define $\psi_{i}: \mathrm{C}_{i} \rightarrow \mathrm{C}$ by letting $\psi_{i}{ }^{+}$be inclusions and $\psi_{i}{ }^{-}(x)=x$ if $x \leq i$ and $\psi_{i}{ }^{-}(x)=i$ for $x>i$. One readily checks that $\left(\psi_{i}: \mathbf{C}_{i} \rightarrow \mathbf{C}\right)_{i>1}$ is a cocone. Consider another cocone defined by $\mathrm{C}^{\prime}:=\left(\mathbb{N}, r^{\prime}, \mathbb{N} \cup\{t\}\right)$, where $r^{\prime}$ extends $r$ with $r^{\prime}(a, t)=1$ for all $a \in \mathbb{N}$. Define $\psi_{i}^{\prime}: \mathrm{C}_{i} \rightarrow \mathrm{C}^{\prime}$ by $\psi_{i}^{\prime+}=\psi_{i}{ }^{+}$and letting $\psi_{i}^{\prime-}$ extend $\psi_{i}^{-}$with $\psi_{i}^{\prime-}(t)=i$. Clearly, $\left(\psi_{i}^{\prime}: \mathbf{C}_{i} \rightarrow \mathbf{C}^{\prime}\right)_{i \geq 1}$ is also a cocone.

Now we can infer that the colimit does not exist. More specifically, suppose $\left(\psi_{i}^{*}: \mathrm{C}_{i} \rightarrow\right.$ $\left.\mathrm{C}^{*}\right)_{i \geq 1}$ with $\mathrm{C}^{*}=\left(A^{*}, r^{*}, X^{*}\right)$ were a colimit. First consider a mediating map $\psi^{\prime}: \mathrm{C}^{*} \rightarrow \mathrm{C}^{\prime}$. We have $\psi^{\prime-}(t)=x^{*}$ for some $x^{*} \in X^{*}$. Then for each $a^{*} \in A^{*}$ we obtain $r^{*}\left(a^{*}, x^{*}\right)=$ $r^{*}\left(a^{*}, \psi^{\prime-}(t)\right)=r^{\prime}\left(\psi^{\prime+}\left(a^{*}\right), t\right)=1$, thus $r^{*}\left(-, x^{*}\right)=1$. Next consider a mediating map $\psi: \mathrm{C}^{*} \rightarrow \mathrm{C}$. Since $\psi^{-}: \mathbb{N} \rightarrow X^{*}$ must be onto, $\psi^{-}(n)=x^{*}$ for some $n \in \mathbb{N}$. We obtain $0=r(n+1, n)=r_{n+1}(n+1, n)=r_{n+1}\left(n+1, \psi_{n+1}^{*}{ }^{-}\left(x^{*}\right)\right)=r^{*}\left(\psi_{n+1}^{*}{ }^{+}(n+1), x^{*}\right)=1$, a contradiction.

Subsequently we will show that particular $\omega$-sequences of Chu spaces do have colimits. For this we provide a generic construction. It is the standard construction in the category of sets, assimilated into the context of Chu spaces. We phrase it explicitly since we will often refer to it.

Construction 3.2. Let $\left(\mathrm{C}_{i}, \varphi_{i}\right)_{i \geq 1}$ be an $\omega$-sequence of Chu spaces where $\mathrm{C}_{i}=\left(A_{i}, r_{i}, X_{i}\right)$ and $\varphi_{i}^{+}: A_{i} \rightarrow A_{i+1}$ is the inclusion mapping, for each $i \geq 1$. Consider $\mathrm{C}:=(A, r, X)$ where

$$
\begin{aligned}
A & :=\bigcup_{i \geq 1} A_{i}, \\
X & :=\left\{\left(x_{j}\right)_{j \geq 1} \mid \forall j \geq 1, x_{j} \in X_{j} \& \varphi_{j}^{-}\left(x_{j+1}\right)=x_{j}\right\}, \\
r\left(a,\left(x_{j}\right)_{j \geq 1}\right) & :=r_{i}\left(a, x_{i}\right) \text { if } a \in A_{i}(i \geq 1) .
\end{aligned}
$$

Subsequently, we will denote a sequence $\left(x_{j}\right)_{j \geq 1} \in X$ often by $\tilde{x}$.

For each $i \geq 1$, define $\mathrm{C}_{i} \stackrel{\psi_{i}}{\longrightarrow} \mathrm{C}$ by $\psi_{i}^{+}(a):=a$ and $\psi_{i}^{-}(\tilde{x}):=x_{i}$ for all $a \in A_{i}$ and $\tilde{x} \in X$.

In Construction 3.2, observe that possibly $X=\emptyset$. Note that the relation $r$ is well-defined since if $i \geq 1$ and $a \in A_{i}$, then $x_{i}=\varphi_{i}^{-}\left(x_{i+1}\right)$ so $r_{i+1}\left(a, x_{i+1}\right)=r_{i}\left(a, x_{i}\right)$; inductively we obtain $r_{j}\left(a, x_{j}\right)=r_{i}\left(a, x_{i}\right)$ for each $j>i$.

Clearly, $\psi_{i}$ is a morphism. Then we have, for each $\tilde{x} \in X$,

$$
\left(\varphi_{i}^{-} \circ \psi_{i+1}^{-}\right)(\tilde{x})=\varphi_{i}^{-}\left(x_{i+1}\right)=x_{i}=\psi_{i}^{-}(\tilde{x})
$$

and for any $a \in A$,

$$
\left(\psi_{i+1}^{+} \circ \varphi_{i}^{+}\right)(a)=a=\psi_{i}^{+}(a)
$$

Therefore, $\psi_{i+1} \circ \varphi_{i}=\psi_{i}$, and $\left(\mathrm{C}_{i} \stackrel{\psi_{i}}{\longrightarrow} \mathrm{C}\right)_{i \geq 1}$ is indeed a cocone. We note:

Proposition 3.3. If an $\omega$-sequence $\left(\mathrm{C}_{i}, \varphi_{i}\right)_{i \geq 1}$ in $\mathbf{i C}$ has a colimit, then this colimit is provided, up to isomorphism, by the cocone $\left(\mathrm{C}_{i} \stackrel{\psi_{i}}{\longrightarrow} \mathrm{C}\right)_{i \geq 1}$ of Construction [3.2.

Proof. Let $\left(\mathrm{C}_{i}, \varphi_{i}\right)_{i \geq 1}$ have a colimit $\left(\mathrm{C}_{i} \stackrel{\psi_{i}^{\prime}}{\longrightarrow} \mathrm{C}^{\prime}\right)_{i \geq 1}$ in $\mathbf{i C}$ where $\mathrm{C}^{\prime}=\left(A^{\prime}, r^{\prime}, X^{\prime}\right)$. By Proposition 2.2(1), the mappings $\varphi_{i}^{+}$are injective and the mappings $\varphi_{i}^{-}$are surjective, and we may assume the $\varphi_{i}^{+}$s to be inclusions. Now construct $\mathrm{C}=(A, r, X)$ and $\psi_{i}: \mathrm{C}_{i} \rightarrow$ $\mathrm{C}(i \geq 1)$. as in Construction 3.2. We claim that each $\psi_{i}(i \geq 1)$ is a morphism in iC. By 
Proposition 2.2(1), it remains to show that $\psi_{i}^{-}$is onto. Using that the $\varphi_{j}^{-} \mathrm{s}$ are onto, for any $x_{i} \in X_{i}$ we can easily find $\tilde{x} \in X$ with $x_{i}=\psi_{i}^{-}(\tilde{x})$.

Since $\mathbf{C}^{\prime}$ is the colimit, there is a unique $\psi: \mathbf{C}^{\prime} \rightarrow \mathbf{C}$ in $\mathbf{i C}$ such that $\psi \circ \psi_{i}^{\prime}=\psi_{i}$ for all $i \geq 1$. Then $\psi^{+}: A^{\prime} \rightarrow A$ is injective. If $a \in A_{i}(i \geq 1)$, then $a=\psi_{i}^{+}(a)=\psi^{+} \circ \psi_{i}^{\prime+}(a)$ and $\psi_{i}^{\prime+}(a) \in A^{\prime}$, so $\psi^{+}$is onto. Further, $\psi^{-}: X \rightarrow X^{\prime}$ is onto, and we claim that $\psi^{-}$ is injective. Let $\tilde{x}, \tilde{y} \in X$ with $\psi^{-}(\tilde{x})=\psi^{-}(\tilde{y})$. For each $i \geq 1$, then $x_{i}=\psi_{i}^{-}(\tilde{x})=$ $\psi_{i}^{\prime-} \circ \psi^{-}(\tilde{x})=\psi_{i}^{\prime-} \circ \psi^{-}(\tilde{y})=\psi_{i}^{-}(\tilde{y})=y_{i}$, showing $\tilde{x}=\tilde{y}$. Hence $\psi$ is an isomorphism.

In contrast to Theorem 3.1 we have the following.

Theorem 3.4. Colimits exist in $\mathbf{i E , ~ a s ~ g i v e n ~ b y ~ C o n s t r u c t i o n ~ 3 . 2 . ~}$

Proof. Let $\left(\mathrm{C}_{i}, \varphi_{i}\right)_{i \geq 1}$ be an $\omega$-sequence in $\mathbf{i E}$ where $\mathrm{C}_{i}=\left(A_{i}, r_{i}, X_{i}\right)$ for each $i \geq 1$. By Proposition 2.2(2), the mappings $\varphi_{i}^{+}$are injective, and we may assume the $\varphi_{i}^{+} \mathrm{s}$ to be inclusions. Now construct $\mathrm{C}=(A, r, X)$ and $\psi_{i}: \mathrm{C}_{i} \rightarrow \mathrm{C}(i \geq 1)$ as in Construction 3.2. We claim that $C$ is extensional. Let $\tilde{x}, \tilde{y} \in X$ and assume that $r(-, \tilde{x})=r(-, \tilde{y})$. We need to show that $\tilde{x}=\tilde{y}$. Indeed, let $i \geq 1$ and choose any $a \in A_{i}$. Then $r_{i}\left(a, x_{i}\right)=r(a, \tilde{x})=$ $r(a, \tilde{y})=r_{i}\left(a, y_{i}\right)$. So $r_{i}\left(-, x_{i}\right)=r_{i}\left(-, y_{i}\right)$ and thus $x_{i}=y_{i}$ as $\mathrm{C}_{i}$ is extensional. Hence $\tilde{x}=\tilde{y}$, and $\mathrm{C}$ is extensional.

For universality, let $\left(C_{i} \stackrel{\psi_{i}^{\prime}}{\longrightarrow} C^{\prime}\right)_{i \geq 1}$ be a cocone, where $C^{\prime}=\left(A^{\prime}, r^{\prime}, X^{\prime}\right)$. Define $\psi$ : $\mathrm{C} \rightarrow \mathrm{C}^{\prime}$ by letting $\psi^{+}: A \rightarrow A^{\prime}$ be such that $\psi^{+}(a):=\psi_{i}^{\prime+}(a)$ if $a \in A_{i}$, and letting $\psi^{-}: X^{\prime} \rightarrow X$ be given as $\psi^{-}\left(x^{\prime}\right):=\left(\psi_{m}^{\prime-}\left(x^{\prime}\right)\right)_{m \geq 1}$. Then $\psi^{+}$is well-defined because for any $1 \leq i<j, \psi_{j}^{\prime+}(a)=\psi_{i}^{\prime+}(a)$; also $\psi^{-}$is well-defined because for any $j \geq 1$, $\varphi_{j}^{-}\left(\psi_{j+1}^{\prime-}\left(x^{\prime}\right)\right)=\psi_{j}^{\prime-}\left(x^{\prime}\right)$, and hence the sequence $\left(\psi_{m}^{\prime-}\left(x^{\prime}\right)\right)_{m \geq 1}$ belongs to $X$. Further, $\psi$ is a morphism. We have $\psi \circ \psi_{i}=\psi_{i}^{\prime}$ for all $i \geq 1$ because $\psi^{+}\left(\psi_{i}^{+}(a)\right)=\psi^{+}(a)=\psi_{i}^{\prime}(a)$, and $\psi_{i}^{-}\left(\psi^{-}\left(x^{\prime}\right)\right)=\psi_{i}^{-}\left(\left(\psi_{j}^{\prime-}\left(x^{\prime}\right)\right)_{j \geq 1}\right)=\psi_{i}^{\prime-}\left(x^{\prime}\right)$ for all $a \in A_{i}$ and $x^{\prime} \in X^{\prime}$, by definitions.

The mediating morphism $\psi$ is a morphism in $\mathbf{i E}$ because $\psi^{+}$is injective, and by Prop. 2.2(2), it is monic. The mediating morphism is unique because its values are fixed by the commutativity requirements of the colimit diagram.

We now consider the biextensional case. In order to avoid potential confusion of terminology, we call a Chu space $\mathrm{C}:=(A, r, X)$ with finite $A$ and $X$ a finite Chu structure. Finite objects in categorical terms will be studied in the next section, as we will learn that finite objects and finite Chu structures do not always agree.

The proof of the following result involves a typical König's-lemma argument for finite $\mathrm{Chu}$ structures which we will encounter again later.

Theorem 3.5. Colimits exist in $\mathbf{i B}$ for $\omega$-sequences of finite Chu structures. They do not exist in general for $\omega$-sequences of arbitrary (non-finite) Chu structures in $\mathbf{i B}$. If for an $\omega$-sequence in $\mathbf{i B}$ there is a cocone to some Chu space in $\mathbf{i B}$, then the sequence has a colimit in $\mathbf{i B}$.

Proof. The proof is similar to that of Theorem 3.4, except that we need to show that in case the $\mathrm{C}_{i}$ constitute a sequence composed of finite biextensional structures, $\mathrm{C}:=(A, r, X)$ is biextensional as well. Since extensionality is already shown to be preserved by this limit structure, it remains to show that separability is preserved.

Suppose all $\mathrm{C}_{i} \mathrm{~s}$ are separable. Let $a, b \in A$ with $r(a,-)=r(b,-)$. Let $m \geq 1$ be minimal with $a, b \in A_{m}$. We claim that $r_{m}(a,-)=r_{m}(b,-)$ in $\mathrm{C}_{m}$. The separability of $\mathrm{C}_{m}$ then gives us $a=b$. 
Suppose $r_{m}(a,-) \neq r_{m}(b,-)$. Then for any $i \geq m$ we have $r_{i}(a,-) \neq r_{i}(b,-)$. For each element $x_{i} \in X_{i}$ such that $r_{i}\left(a, x_{i}\right) \neq r_{i}\left(b, x_{i}\right)$ let $s\left(x_{i}\right)=\left(y_{j}\right)_{1 \leq j \leq i}$ be the sequence defined inductively by $y_{i}=x_{i}$ and $y_{j-1}=\varphi_{j-1}^{-}\left(y_{j}\right)$ for each $j=i, i-1, \ldots, 2$. Consider the set

$$
T:=\left\{s\left(x_{i}\right) \mid i \geq m\right\} .
$$

Clearly, $T$ is an infinite set. Consider elements of $T$ as nodes, and an edge from $s\left(x_{i}\right)$ to $s\left(x_{j}\right)$ exists if $j=i+1$, and $s\left(x_{i+1}\right)$ is an extension of $s\left(x_{i}\right)$, that is, $x_{i}=\varphi_{i}^{-}\left(x_{i+1}\right)$. Observe that if $i \geq m, x_{i+1} \in X_{i+1}$, and $x_{i}=\varphi_{i}^{-}\left(x_{i+1}\right)$, then $r_{i}\left(a, x_{i}\right) \neq r_{i}\left(b, x_{i}\right)$ iff $r_{i+1}\left(a, x_{i+1}\right) \neq r_{i+1}\left(b, x_{i+1}\right)$. So, in this case $s\left(x_{i}\right)$ is defined iff $s\left(x_{i+1}\right)$ is defined, and then they are connected by an edge. Since each $X_{i}$ is finite, $T$ is a finite branching, infinite tree. By König's Lemma, this tree has an infinite branch, say, $\left(s\left(x_{i}\right)\right)_{i \geq 1}$. Consequently, $\varphi_{i}^{-}\left(x_{i+1}\right)=x_{i}$ for all $i \geq 1$. Clearly, by the construction above, we have $\tilde{x}=\left(x_{i}\right)_{i \geq 1} \in X$ and $r(a, \tilde{x}) \neq r(b, \tilde{x})$, a contradiction.

For the second part of the theorem, we construct a counterexample as follows. Let $\mathrm{C}_{i}:=\left(\mathbb{N}, r_{i}, \mathbb{N}\right)$ for $i \geq 1$, with $r_{i}(a, x)=1$ iff $(x+i-1) \bmod a=0$ and $r_{i}(a, x)=0$ otherwise. For example, $r_{1}$ is displayed below as a countable matrix, which will be referred to as $\mathbb{M}$ for future reference.

\begin{tabular}{|llllllll|}
\hline 1 & 1 & 1 & 1 & 1 & 1 & 1 & $\cdots$ \\
0 & 1 & 0 & 1 & 0 & 1 & 0 & $\cdots$ \\
0 & 0 & 1 & 0 & 0 & 1 & 0 & $\cdots$ \\
0 & 0 & 0 & 1 & 0 & 0 & 0 & $\cdots$ \\
0 & 0 & 0 & 0 & 1 & 0 & 0 & $\cdots$ \\
0 & 0 & 0 & 0 & 0 & 1 & 0 & $\cdots$ \\
0 & 0 & 0 & 0 & 0 & 0 & 1 & $\cdots$ \\
& & & & $\vdots$ & & & \\
\hline
\end{tabular}

Intuitively, $r_{i}$ is obtained by starting from the countable matrix $r_{1}$ from the $i$-th column. Clearly, $C_{i}$ is biextensional. The morphism $\varphi_{i}: \mathrm{C}_{i} \rightarrow \mathrm{C}_{i+1}$ is defined by $\varphi_{i}^{+}:=i d_{\mathbb{N}}$, and $\varphi_{i}^{-}(x)=x+1$. Then,

$$
r_{i+1}\left(\varphi_{i}^{+}(a), x\right)=r_{i+1}(a, x)=r_{i}(a, x+1)=r_{i}\left(a, \varphi_{i}^{-}(x)\right),
$$

and so $\varphi_{i}$ is indeed a Chu morphism for each $i \geq 1$.

Now assume that $\left(\psi_{i}: \mathrm{C}_{i} \rightarrow \mathrm{C}\right)_{i \geq 1}$ is a cocone where $\mathrm{C}=(A, r, X)$. We show that then $X=\emptyset$. Suppose there is $x \in X$. Then, for any $i \geq 1, \psi_{i}^{-}(x)=\varphi_{i}^{-}\left(\psi_{i+1}^{-}(x)\right)=\psi_{i+1}^{-}(x)+1$ by the definition of $\varphi_{i}^{-}$. Inductively, we have $\psi_{n+1}^{-}(x)=\psi_{1}^{-}(x)-n$ for all $n \geq 1$. But $\psi_{n+1}^{-}(x) \in \mathbb{N}$ for all $n \geq 1$, a contradiction. Hence $X$ is empty. But then $\mathrm{C}$ is not separable.

For the last part of the theorem, let $\left(\mathrm{C}_{i}, \varphi_{i}\right)_{i \geq 1}$ be an $\omega$-sequence in $\mathbf{i B}$ with some cocone $\left(\mathrm{C}_{i} \stackrel{\psi^{\prime} i}{\longrightarrow} \mathrm{C}^{\prime}\right)_{i \geq 1}$ in iB. By Theorem 3.4 , the sequence $\left(\mathrm{C}_{i}, \varphi_{i}\right)_{i \geq 1}$ has a colimit $\left(\mathrm{C}_{i} \stackrel{\psi_{i}}{\longrightarrow} \mathrm{C}\right)_{i \geq 1}$ in $\mathbf{i E}$. We claim that this is also the colimit of the sequence in $\mathbf{i B}$. Now there is a morphism $\psi: \mathrm{C} \rightarrow \mathrm{C}^{\prime}$ in $\mathbf{i E}$ making the diagram commute. We show that $\mathrm{C}$ is separable. Let $\mathrm{C}=$ $(A, r, X)$ and $\mathrm{C}^{\prime}=\left(A^{\prime}, r^{\prime}, X^{\prime}\right)$. Choose any $a, b \in A$ with $r(a,-)=r(b,-)$. For any $x^{\prime} \in X^{\prime}$ we have $r^{\prime}\left(\psi^{+}(a), x^{\prime}\right)=r\left(a, \psi^{-}\left(x^{\prime}\right)\right)=r\left(b, \psi^{-}\left(x^{\prime}\right)\right)=r^{\prime}\left(\psi^{+}(b), x^{\prime}\right)$, so $\psi^{+}(a)=\psi^{+}(b)$ since $\mathrm{C}^{\prime}$ is biextensional, and $a=b$ as $\psi^{+}$is injective.

Hence $\mathrm{C}$ is biextensional, and by Proposition 2.2(2) and (3), the morphisms $\psi_{i}: \mathrm{C}_{i} \rightarrow \mathrm{C}$ $(i \geq 1)$ belong to $\mathbf{i B}$, and our claim follows. 
It is informative to think about the example given in the proof of Theorem 3.1. By the colimit construction, $\mathbf{C}^{\prime}=\left(\mathbb{N}, r^{\prime}, \mathbb{N} \cup\{t\}\right)$ is the colimit both in $\mathbf{i E}$ and in $\mathbf{i B}$. There is indeed a monic morphism $\psi$ from $\mathrm{C}^{\prime}$ to $\mathbf{C}=(\mathbb{N}, r, \mathbb{N})$, where $\psi^{+}$is the identity (injection), and $\psi^{-}$is the inclusion (but not onto).

Also note that even though Theorem 3.4 confirms that colimit always exists for extensional Chu spaces, the counterexample for Theorem 3.5 shows that colimits for infinite structures may have weird behaviors with unintended effects. This invites us to look more into objects constructed as colimits of finite structures, in the next sections.

\section{Finite oBJECTS}

In studying patterns of approximation in Chu spaces, finite objects play an important role since they serve as the basis of approximation. In most cases, one expects finite objects to correspond to finite structures, objects whose constituents are finite sets. In categorical terms, finite objects are captured using colimits in a standard way, and the notion of "approximation" is captured by monic morphisms. Therefore, we work with categories $\mathbf{i C}, \mathbf{i E}$, and iB. However, since Prop. 2.2 indicates that what counts as monic morphisms depends on extensionality, the existence of colimits and the characterization of finite objects are not straightforward set-theoretic generalizations obtained by treating each component of Chu spaces separately.

We give a characterization of the finite objects of iC. Surprisingly, not all finite structures in $\mathbf{i C}$ are finite objects; finite objects are characterized as extensional structures with finite object set instead. The following definition is phrased in $\mathbf{i C}$; but as a general categorical concept it can be made explicit in $\mathbf{i E}$ and $\mathbf{i B}$ as well, and we do not repeat this here.

Definition 4.1. An object $\mathrm{F}$ of $\mathbf{i C}$ is finite if for every $\omega$-sequence $\left(C_{i}, \varphi_{i}\right)_{i \geq 1}$ of $\mathrm{Chu}$ spaces having a colimit, for every morphism $\varphi: \mathrm{F} \rightarrow \operatorname{colim}_{i}\left(\mathrm{C}_{i}, \varphi_{i}\right)$ in $\mathbf{i C}$ there exist $i \geq 1$ and a morphism $\psi: \mathrm{F} \rightarrow \mathrm{C}_{i}$ such that the diagram

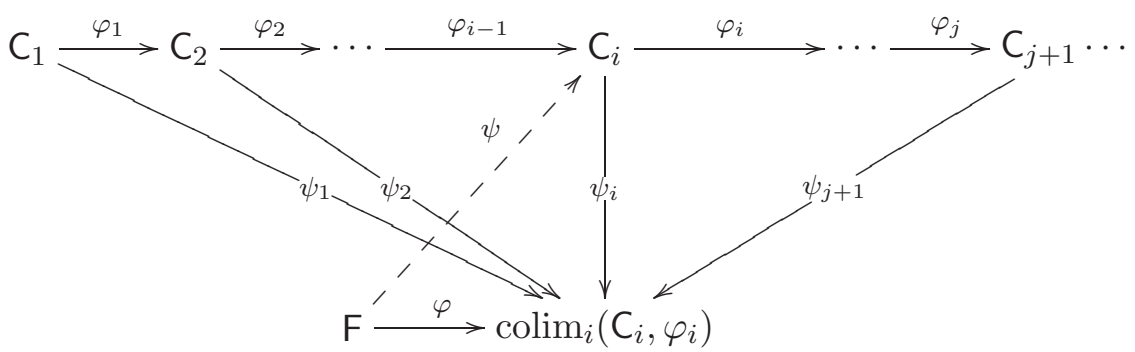

commutes, i.e., $\psi: \mathrm{F} \rightarrow \mathrm{C}_{i}$ is such that $\varphi=\psi_{i} \circ \psi$.

If $\Sigma$ is finite and $\mathbf{F}=(A, r, X)$ is a finite object in $\mathbf{i C}$, one can show that both $A$ and $X$ are finite sets, i.e. $\mathrm{F}$ is a finite $\mathrm{Chu}$ space. However, somewhat surprisingly (at least to us), the converse does not hold, as already simple examples show, see Example 4.2 below. The following result characterizes the finite objects of $\mathbf{i C}$.

Theorem 4.1. An object $\mathrm{F}=(B, s, Y)$ is finite in $\mathbf{i C}$ iff $B$ is finite and $\mathrm{F}$ is extensional. In this case, $|Y| \leq|\Sigma|^{|B|}$; in particular, if $\Sigma$ is finite, so is $Y$. 
Proof. (Only if.) Suppose $\mathbf{F}=(B, s, Y)$ is a finite object of $\mathbf{i C}$ as in Definition 4.1. If $B$ is infinite, then we can write $B=A_{1} \cup\left\{a_{i} \mid i \geq 1\right\}$, where $A_{1} \cap\left\{a_{i} \mid i \geq 1\right\}=\emptyset$. Let $\mathrm{C}_{i}:=\left(A_{i}, s_{i}, Y\right)$, where $A_{i}=A_{1} \cup\left\{a_{1}, \ldots, a_{i}\right\}$ and $s_{i}$ is $s$ restricted to the product $A_{i} \times Y$. It can then be checked that $\left(\psi_{i}: \mathrm{C}_{i} \rightarrow \mathrm{F}\right)_{i \geq 1}$ is the colimit of the $\omega$-sequence $\left(\mathrm{C}_{i}, \varphi_{i}\right)_{i \geq 1}$, with $\varphi_{i}^{+}, \psi_{i}^{+}$inclusion and $\varphi_{i}^{-}, \psi_{i}^{-}$identity for all $i \geq 1$. We have $\left(i d_{B}, i d_{Y}\right): \mathrm{F} \rightarrow \mathrm{F}$ a morphism. Since $\mathrm{F}$ is a finite object, there exist an $i \geq 1$ and $\psi: \mathrm{F} \rightarrow \mathrm{C}_{i}$, such that $i d_{B}=\psi_{i}^{+} \circ \psi^{+}$. Then $a_{i+1}=\psi_{i}^{+} \circ \psi^{+}\left(a_{i+1}\right)=\psi^{+}\left(a_{i+1}\right) \in A_{i}$, a contradiction. Therefore, $B$ must be finite.

Next we show that $\mathrm{F}$ is extensional. Suppose there are $y_{1}, y_{2} \in Y$ with $y_{1} \neq y_{2}$ and $s\left(-, y_{1}\right)=s\left(-, y_{2}\right)$. Put $Y^{\prime}=Y \backslash\left\{y_{1}, y_{2}\right\}$. We may assume that $Y^{\prime} \cap \mathbb{N}=\emptyset$. Now let $\mathrm{C}_{i}=$ $\left(B, s_{i}, Y_{i}\right)$ with $Y_{i}=Y^{\prime} \cup\{1, \ldots, i\}$ and $\mathrm{C}=\left(B, s^{\prime}, Y^{\prime} \cup \mathbb{N}\right)$. Put $s^{\prime}(b, y)=s_{i}(b, y)=s(b, y)$ for each $y \in Y^{\prime}$ and $s^{\prime}(b, j)=s_{i}(b, j)=s\left(b, y_{1}\right)$ for each $1 \leq j \leq i \in \mathbb{N}$ and $b \in B$. We let $\varphi_{i}^{+}$be the identities, and $\varphi_{i}^{-}$leave everything unchanged except $\varphi_{i}^{-}(i+1)=1$. Also, let $\psi_{i}^{+}$be the identity map, $\psi_{i}^{-}(j)=j$ if $j \leq i$ or $j \in Y^{\prime}$, and $\psi_{i}^{-}(j)=1$ if $j>i$. Then the $\omega$-sequence $\left(\mathrm{C}_{i}, \varphi_{i}\right)_{i \geq 1}$ has $\left(\mathrm{C}_{i} \stackrel{\psi_{i}}{\longrightarrow} \mathrm{C}\right)_{i \geq 1}$ as its colimit. Now define $\varphi: \mathrm{F} \rightarrow \mathrm{C}$ with $\varphi^{+}=i d_{B}, \varphi^{-}$mapping odd numbers to $y_{1}$, even numbers to $y_{2}$, and leaving elements in $Y^{\prime}$ unchanged. Since $\mathbf{F}$ is finite, there are an $i \geq 1$ and a morphism $\psi: \mathbf{F} \rightarrow \mathbf{C}_{i}$ such that $\varphi=\psi_{i} \circ \psi$. Then $\left(\psi^{-} \circ \psi_{i}^{-}\right)(i+1)=\psi^{-}(1)=\left(\psi^{-} \circ \psi_{i}^{-}\right)(i+2)$, contradicting the assumption that $\varphi^{-}(i+1) \neq \varphi^{-}(i+2)$.

(If) Suppose $\varphi: \mathrm{F} \rightarrow \operatorname{colim}_{i}\left(\mathrm{C}_{i}, \varphi_{i}\right)$ in $\mathbf{i C}$, where $B$ is finite, $\mathrm{F}$ is extensional and $\mathrm{C}_{i}=$ $\left(A_{i}, r_{i}, X_{i}\right)(i \geq 1)$. Since the colimit of an $\omega$-sequence is unique up to isomorphism, we may assume that all $\varphi_{i}^{+}$s are inclusions and that $\operatorname{colim}_{i}\left(C_{i}, \varphi_{i}\right)$ is the structure $\left(\mathrm{C}_{i} \stackrel{\psi_{i}}{\longrightarrow} \mathrm{C}\right)_{i \geq 1}$ with $\mathrm{C}=(A, r, X)$ given in Construction 3.2. We can further assume that $\varphi^{+}$is an inclusion by renaming the elements of $B$. As $B$ is a finite set, $B \subseteq A=\bigcup_{i} A_{i}$ implies that $B \subseteq A_{m}$ for some $m \geq 1$. Now define $\psi^{-}: X_{m} \rightarrow Y$ by $q \mapsto \varphi^{-}(x)$ if and only if $x \in X$ satisfies $q=\psi_{m}^{-}(x)$.

We show that $\psi^{-}$is a function. For this, let $x, x^{\prime} \in X$ such that $\psi_{m}^{-}(x)=\psi_{m}^{-}\left(x^{\prime}\right)=q$. Observe that $B \subseteq A_{m} \subseteq A$. For any $b \in B$, we have

$$
\begin{aligned}
s\left(b, \varphi^{-}(x)\right) & =r(b, x) & & \left(\varphi^{+} \text {is inclusion }\right) \\
& =r_{m}\left(b, \psi_{m}^{-}(x)\right) & & \left(\psi_{m}^{+} \text {is inclusion }\right) \\
& =r_{m}\left(b, \psi_{m}^{-}\left(x^{\prime}\right)\right) & & \left(\psi_{m}^{-}(x)=\psi_{m}^{-}\left(x^{\prime}\right)\right) \\
& =r\left(b, x^{\prime}\right) & & \left(\psi_{m}^{+} \text {is inclusion }\right) \\
& =s\left(b, \varphi^{-}\left(x^{\prime}\right)\right) & & \left(\varphi^{+} \text {is inclusion }\right)
\end{aligned}
$$

Therefore, $s\left(-, \varphi^{-}(x)\right)=s\left(-, \varphi^{-}\left(x^{\prime}\right)\right)$ and by extensionality, $\varphi^{-}(x)=\varphi^{-}\left(x^{\prime}\right)$.

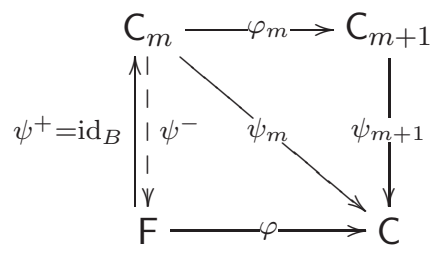

(where $\mathrm{C}=\operatorname{colim}_{i}\left(\mathrm{C}_{i}, \varphi_{i}\right)$ )

We check that $\psi=\left(\operatorname{id}_{B}, \psi^{-}\right): \mathrm{F} \rightarrow \mathrm{C}_{m}$ is a Chu morphism. Indeed, for any $b \in B$ and $x_{m} \in X_{m}$, we have 


$$
\begin{array}{rlr}
r_{m}\left(\psi^{+}(b), x_{m}\right) & =r_{m}\left(b, x_{m}\right) & \left(\psi^{+}=\mathrm{id}_{B}\right) \\
& =r_{m}\left(b, \psi_{m}^{-}(x)\right) & \left(\psi_{m}^{-} \text {onto; } \psi_{m}^{-}(x)=x_{m}\right) \\
& =r\left(\psi_{m}^{+}(b), x\right) & \left(\psi_{m}: \mathrm{C}_{m} \rightarrow \mathrm{C}\right) \\
& =r(b, x) & \left(\psi_{m}^{+}=\mathrm{id}_{A_{m}}\right) \\
& =r\left(\varphi^{+}(b), x\right) & \left(\varphi^{+}=\operatorname{id}_{B}\right) \\
& =s\left(b, \varphi^{-}(x)\right) & (\varphi: \mathrm{F} \rightarrow \mathrm{C}) \\
& =s\left(b,\left(\psi^{-} \circ \psi_{m}^{-}\right)(x)\right) & \left(\psi^{-} \circ \psi_{m}^{-}=\varphi^{-}\right) \\
& =s\left(b, \psi^{-}\left(x_{m}\right)\right) & \left(\psi_{m}^{-}(x)=x_{m}\right)
\end{array}
$$

as required. Since $\varphi^{-}$is onto, $\psi^{-}$is onto, and $\psi$ is monic. Finally, we have $\psi^{-} \circ \psi_{m}^{-}=\varphi^{-}$ since for any $x \in X$, by the definition of $\psi^{-}, \psi^{-}\left(\psi_{m}^{-}(x)\right)=\varphi^{-}(x)$. Hence $\varphi=\psi_{m} \circ \psi$, and $F$ is shown to be finite.

Finally, let $\Sigma^{B}$ denote the set of all functions from $B$ into $\Sigma$. Note that $s(-, y) \in \Sigma^{B}$ for each $y \in Y$, and if $\mathrm{F}$ is extensional, the mapping $y \mapsto s(-, y)$ provides an injection of $Y$ into $\Sigma^{B}$, showing $|Y| \leq\left|\Sigma^{B}\right|=|\Sigma|^{|B|}$ by cardinal arithmetic.

Next we give two examples to illustrate Theorem 4.1 .

Example 4.2. Let $\Sigma=\{0,1\}$ and $F:=(\{\star\}, r,\{1,2\})$, a finite Chu space. If $r(\star, 1)=$ $r(\star, 2)=0$, then $F$ is not extensional and thus, by Theorem 4.1, not a finite object of iC. To see this more explicitly, one can construct a sequence $\left(C_{i}, \varphi_{i}\right)_{i \geq 1}$ as in the proof of Theorem 4.1, with $Y^{\prime}=\emptyset$.

Example 4.3. Only in this example, let $\Sigma$ be an arbitrary (possibly infinite) set, and let $\mathrm{F}=(\{\star\}, r, \Sigma)$ with $r(\star, \sigma)=\sigma$ for each $\sigma \in \Sigma$. Then $\mathrm{F}$ is extensional, and by Theorem 4.1, $\mathrm{F}$ is a finite object of $\mathbf{i C}$. Trivially, if $\Sigma$ is infinite, $\mathrm{F}$ is not a finite Chu space.

Theorem 4.4. In the category $\mathbf{i E}$, if $\mathrm{F}=(B, s, Y)$ is finite then $B$ is finite.

An independent proof is needed even though we follow a similar path as the proof of Theorem 4.1. Not only should we make sure that the Chu spaces involved are all extensional, but also the monic morphisms are characterized differently. These entail non-trivial modifications from the proof of Theorem 4.1.

Proof. Suppose $\mathbf{F}=(B, s, Y)$ is a finite object in $\mathbf{i E}$. Suppose $B$ is infinite. Then we can write $B=A_{1} \cup\left\{a_{i} \mid i \geq 1\right\}$, where $A_{1} \cap\left\{a_{i} \mid i \geq 1\right\}=\emptyset$. Fix $c \in Y$. Let $\mathrm{C}_{i}:=$ $\left(A_{1} \cup\left\{a_{1}, \ldots, a_{i}\right\}, r_{i}, X_{i}\right)$, where $X_{i}=\{c\}$ and $r_{i}$ is $s$ restricted to the product $\left(A_{1} \cup\right.$ $\left.\left\{a_{1}, \ldots, a_{i}\right\}\right) \times\{c\}$. Clearly, all $\mathrm{C}_{i}$ s are extensional. For morphisms $\varphi_{i}: \mathrm{C}_{i} \rightarrow \mathrm{C}_{i+1}$, define $\varphi_{i}^{+}$ as inclusions, and $\varphi_{i}^{-}: X_{i+1} \rightarrow X_{i}$ the identity. By Theorem 3.4 , the colimit $\left(\mathrm{C}_{i} \stackrel{\psi_{i}}{\longrightarrow} \mathrm{C}\right)_{i \geq 1}$ with $\mathrm{C}=(A, r, X)$ of the sequence $\left(\mathrm{C}_{i}, \varphi_{i}\right)_{i \geq 1}$ exists, and can be taken as the one given in Construction 3.2. Since each $X_{i}$ is a singleton, $X$ is a singleton as well. Thus we may assume $A=B$. With $\varphi^{+}$identity and $\varphi^{-}$inclusion, we obtain a monic morphism $\varphi$ from $\mathrm{F}$ to $\mathrm{C}$. Hence there is a monic morphism $\psi$ from $\mathrm{F}$ to some $\mathrm{C}_{i}$ which makes the required diagram commute. But then $\varphi^{+}\left(a_{i+1}\right)=\psi_{i}^{+}\left(\psi^{+}\left(a_{i+1}\right)\right) \neq a_{i+1}$, a contradiction.

The converse of Theorem 4.4 is not true. To show this, we adapt the counterexample for the second part of Theorem 3.5 as follows. Let $C_{i}:=\left(\mathbb{N}, r_{i}, \mathbb{N} \cup\{c\}\right)$, with $r_{i}(a, x)=1$ iff $(x+i-1) \bmod a=0$ for $a, x \in \mathbb{N}$, and $r_{i}(-, c)=1$. Intuitively, $r_{i}$ is obtained by starting from the countable matrix $r_{1}$ from the $i$-th column. The morphism $\varphi_{i}: C_{i} \rightarrow C_{i+1}$ is defined by $\varphi_{i}^{+}:=i d_{\mathbb{N}}$, and $\varphi_{i}^{-}(x)=x+1$, but we keep $c$ constant. Then, the colimit of this 
sequence is $\mathrm{C}:=(\mathbb{N}, r,\{c\})$, with $r(-, c)=1$. Now let $\mathrm{B}:=(\{1,2\}, s,\{c\})$, with inclusion and identity paired to form a morphism $\varphi$ from B to C. There cannot be a morphism $\psi$ from $\mathrm{B}$ to any $\mathrm{C}_{i}$, because $\psi^{-}:(\mathbb{N} \cup\{c\}) \rightarrow\{c\}$ cannot be defined, simply because B's column contains two $1 \mathrm{~s}$, and each $r_{i}(2, i)=0$. Hence $B$ is a finite extensional Chu space and thus a finite object of $\mathbf{i C}$ but not of $\mathbf{i E}$.

Definition 4.2. A Chu space $(A, r, X)$ over $\Sigma$ is called discrete, if for any mapping $f: A \rightarrow$ $\Sigma$ there is $x \in X$ with $f=r(-, x)$.

Theorem 4.5. In the category $\mathbf{i E}, \mathrm{F}=(B, s, Y)$ is finite iff $B$ is finite and $\mathrm{F}$ is discrete.

Proof. (Only if). By Theorem 4.4, we know that $B$ is finite. Suppose $\mathbf{F}=(B, s, Y)$ is not discrete. Let $v: B \rightarrow \Sigma$ be such that $v \neq s(-, y)$ for any $y \in Y$. Let $C_{i}:=\left(B \cup \mathbb{N}, r_{i}, Y \cup \mathbb{N}\right)$, with $r_{i}(a, y)=s(a, y)$ for $a \in B$ and $y \in Y$, and $r_{i}(a, x)=1$ iff $(x+i-1) \bmod a=0$ for $a, x \in \mathbb{N}$. Furthermore, for all $a \in \mathbb{N}$ and $y \in Y$, we let $r_{i}(a, y)=0$, and for all $b \in B$ and $x \in \mathbb{N}$, we let $r_{i}(b, x)=v(b)$. Viewed as an infinite matrix, $r_{i}$ is obtained by placing $s$ at the upper-left corner, and the infinite matrixes used in Theorem 3.5 on the lower-right corner. The lower-left corner is filled with zeros, and the upper-right corner is filled with repeated columns duplicating $v$. A rendering of $r_{1}$ is given next, where $\mathbb{O}$ is a $\mathbb{N} \times Y$ matrix of all $0 \mathrm{~s}$, and $\mathbb{M}$ is the countable matrix used in the proof of Theorem 3.5 .

\begin{tabular}{|l|l|l|l|l|l|}
\multicolumn{1}{c|}{} & \multicolumn{3}{c|}{$Y$} & \multicolumn{3}{c|}{$\mathbb{N}$} \\
\hline & \multicolumn{1}{c|}{$B$} & $v$ & $v$ & $v$ & $\cdots$ \\
\hline $\mathbb{N}$ & $\mathbb{O}$ & \multicolumn{4}{|c|}{$\mathbb{M}$} \\
\hline
\end{tabular}

We define morphisms $\varphi_{i}: \mathrm{C}_{i} \rightarrow \mathrm{C}_{i+1}(i \geq 1)$ by letting $\varphi_{i}^{+}=i d_{B \cup \mathbb{N}},\left.\varphi_{i}^{-}\right|_{Y}=i d_{Y}$ and $\varphi_{i}^{-}(x)=x+1$ for each $x \in \mathbb{N}$. One can check that up to isomorphism the colimit of this sequence is $\left(\psi_{i}: \mathrm{C}_{i} \rightarrow \mathrm{C}\right)_{i \geq 1}$ where $\mathbf{C}=\left(B \cup \mathbb{N}, s^{\prime}, Y\right)$ such that $s^{\prime}$ coincides with $s$ on $B \times Y$, and $s^{\prime}(a, y)=0$ for each $a \in \mathbb{N}, y \in Y$; further, $\psi_{i}^{+}=i d_{B \cup \mathbb{N}}$ and $\psi_{i}^{-}=i d_{Y}$. Clearly, $\varphi=\left(i d_{B}, i d_{Y}\right)$ is a monomorphism from $\mathrm{F}$ to $\mathrm{C}$. Since $\mathrm{F}$ is finite, there are $i \geq 1$ and a morphism $\psi: \mathrm{F} \rightarrow \mathrm{C}_{i}$ which make the diagram commute. Then $\psi^{+}=i d_{B}$, and for any $b \in B$ and $x \in \mathbb{N}$ we obtain $r_{i}(b, x)=s\left(b, \psi^{-}(x)\right) \neq v(b)=r_{i}(b, x)$, a contradiction.

(If). We follow a pattern similar to the "if" part for Theorem 4.1. Suppose $\varphi: F \rightarrow$ $\operatorname{colim}_{i}\left(\mathrm{C}_{i}, \varphi_{i}\right)$ in $\mathbf{i E}$, where $\mathbf{F}=(B, s, Y)$ is discrete and $B$ is finite. By Theorem 3.4, we may assume that $\operatorname{colim}_{i}\left(\mathrm{C}_{i}, \varphi_{i}\right)$ is the structure $\mathrm{C}$ given in Construction 3.2. We can further assume that $\varphi^{+}$is an inclusion by renaming its elements. As $B$ is a finite set, $B \subseteq A=\bigcup_{i} A_{i}$ implies that $B \subseteq A_{m}$ for some $m \geq 1$. Since $\mathrm{F}$ is discrete, we can define $\psi^{-}: X_{m} \rightarrow Y$ such that for each $x \in X_{m}, \psi^{-}(x) \in Y$ is such that for all $b \in B, r_{m}(b, x)=s\left(b, \psi^{-}(x)\right)$. This entails that $\left(\mathrm{id}_{B}, \psi^{-}\right): \mathrm{F} \rightarrow \mathrm{C}_{m}$ is a Chu morphism.

To check the condition $\psi^{-} \circ \psi_{m}^{-}=\varphi^{-}$, note that for any $b \in B$ and $x \in X_{m}$, we have

$$
\begin{array}{rlr}
s\left(b, \psi^{-}\left(\psi_{m}^{-}(x)\right)\right) & =r_{m}\left(b, \psi_{m}^{-}(x)\right) & \left(\text { def. of } \psi^{-}\right) \\
& =r\left(\psi_{m}^{+}(b), x\right) & \left(\psi_{m}: \mathrm{C}_{m} \rightarrow \mathrm{C}\right) \\
& =r(b, x) & \left(\psi_{m}^{+}=\mathrm{id}_{A_{m}}\right) \\
& =r\left(\varphi^{+}(b), x\right) & \left(\varphi^{+}=\mathrm{id}_{B}\right) \\
& =s\left(b, \varphi^{-}(x)\right) & (\varphi: \mathrm{F} \rightarrow \mathrm{C})
\end{array}
$$

and by the extensionality of $\mathrm{F}$, we have $\psi^{-}\left(\psi_{m}^{-}(x)\right)=\varphi^{-}(x)$, as required. 
The following easy remark shows that the structure of discrete extensional Chu spaces $\mathrm{C}=(A, r, X)$ is very restricted: it is completely determined, up to isomorphism, by the cardinality of the object set $A$.

Remark 4.6. Let $\mathrm{C}=(A, r, X)$ and $\mathrm{C}^{\prime}=\left(A^{\prime}, r^{\prime}, X^{\prime}\right)$ be two discrete extensional Chu spaces with $|A|=\left|A^{\prime}\right|$. Then $\mathbf{C}$ and $\mathbf{C}^{\prime}$ are isomorphic in $\mathbf{i C}$.

Proof. Choose a bijection $\varphi^{+}: A \rightarrow A^{\prime}$. By the assumption on C, for each $x^{\prime} \in X^{\prime}$ there is a uniquely determined $x \in X$ with $r(-, x)=r^{\prime}\left(-, x^{\prime}\right) \circ \varphi^{+}$. The mapping $\varphi^{-}: X^{\prime} \rightarrow X$ with $\varphi^{-}\left(x^{\prime}\right)=x$ yields a Chu morphism $\varphi=\left(\varphi^{+}, \varphi^{-}\right)$, and $\varphi^{-}$is bijective by the assumption on $\mathrm{C}^{\prime}$.

Almost similar to Theorem 4.5, we have the following. However, an independent proof is needed because the structures used in the proof for Theorem 4.5 are not biextensional, and an extra case arises..

Theorem 4.7. In the category $\mathbf{i B}, \mathrm{F}=(B, s, Y)$ is finite iff $B$ is finite and $\mathrm{F}$ is discrete, or else $B$ is a singleton, $Y=\emptyset$, and $\Sigma$ is finite.

Proof. (If.) In the first case, by Theorem 4.5, $\mathrm{F}$ is finite in $\mathbf{i E}$, and $\mathrm{F}$ is biextensional. Note that a colimit of an $\mathbf{i B}$-sequence taken in $\mathbf{i B}$ coincides with the colimit of this sequence taken in $\mathbf{i E . ~ H e n c e ~} \mathbf{F}$ is finite in $\mathbf{i B}$.

Secondly, assume $B$ is a singleton, $Y=\emptyset$, and $\Sigma$ is finite. Suppose $\varphi: \mathrm{F} \rightarrow \operatorname{colim}_{i}\left(\mathrm{C}_{i}, \varphi_{i}\right)$ in iB. By Theorem 3.5, we may name $\operatorname{colim}_{i}\left(\mathrm{C}_{i}, \varphi_{i}\right)$ as the structure $\mathrm{C}=(A, r, X)$ given in Construction 3.2. Since $\varphi^{-}: X \rightarrow Y$ is a mapping and $Y=\emptyset$, we also obtain that $X=\emptyset$. Thus $A$ is a singleton, since $C$ is biextensional. Hence we may assume that $B=A=A_{i}$ for each $i \geq 1$. Since each $C_{i}$ is biextensional and $\Sigma$ is finite, we obtain that $X_{i}$ is finite, too.

Suppose that $X_{i} \neq \emptyset$ for each $i \geq 1$. For each $i \geq 1$ and each element $x_{i} \in X_{i}$ define the sequence $s\left(x_{i}\right)$ as in the proof of Theorem 3.5, and let again $T=\left\{s\left(x_{i}\right) \mid i \geq 1, x_{i} \in X_{i}\right\}$. Then with the extension order, $T$ is an infinite finite-branching tree since each $X_{i}$ is finite, and therefore $T$ contains, by König's Lemma, an infinite branch. This implies that $X \neq \emptyset$, a contradiction.

Hence we have $X_{i}=\emptyset$ for some $i \geq 1$. Then $\psi_{i}=\left(\varphi^{+}, \emptyset\right): \mathrm{F} \rightarrow \mathrm{C}_{i}$ makes the diagram commute, showing that $\mathrm{F}$ is finite.

(Only if.) First we assume that $Y=\emptyset$. Since $\mathrm{F}$ is biextensional, $B$ must be a singleton, say, $B=\{b\}$. We claim that $\Sigma$ is finite. Suppose $\Sigma$ was infinite. We may assume that $\mathbb{N} \subseteq \Sigma$. We define Chu spaces $C_{i}=\left(A_{i}, r_{i}, X_{i}\right)$ such that $A_{i}=B, X_{i}=\{j \in \mathbb{N} \mid j \geq i\}$ and $r_{i}(b, j)=j$ for each $j \geq i$ and $i \geq 1$. Also, let $\varphi_{i}^{+}$be the identity mapping and $\varphi_{i}^{-}$be the inclusion. Then $\left(\mathrm{C}_{i}, \varphi_{i}\right)_{i \geq 1}$ is an $\omega$-chain in $\mathbf{i B}$ having $\mathrm{F}$ as its colimit. So there exists some $i \geq 1$ such that $\psi_{i}: \mathrm{F} \rightarrow \mathrm{C}_{i}$. In particular, $\psi_{i}^{-}: X_{i} \rightarrow Y$ is a mapping, which contradicts the assumption that $Y=\emptyset$.

Next we assume that $Y \neq \emptyset$ and we show that $\mathrm{F}$ is discrete.

For this, we refine the arguments employed for Theorem 4.5. If $\mathrm{F}=(B, s, Y)$ is not discrete, choose $v: B \rightarrow \Sigma$ such that $v \neq s(-, y)$ for any $y \in Y$. Now choose an infinite set $J$ of size at least $|B \cup Y|$. Put $Y^{\prime}=\bigcup_{j \in J} Y \times\{j\}$; we write $Y_{j}$ for $Y \times\{j\}$ for conciseness.

Let $\mathrm{C}_{i}:=\left(B \cup J \cup \mathbb{N}, r_{i}, Y^{\prime} \cup \mathbb{N}\right)(i \geq 1)$ and $\mathrm{C}=\left(B \cup J \cup \mathbb{N}, r, Y^{\prime}\right)$ where $r$ and $r_{i}(i \geq 1)$ are defined as follows:

On each $B \times Y_{j}(j \in J)$, define $r$ and $r_{i}$ precisely as $s$ in $\mathrm{F}$ on $B \times Y$. Next, choose a bijection $\pi: J \cup \mathbb{N} \rightarrow Y^{\prime}$. On $(J \cup \mathbb{N}) \times Y^{\prime}$ define $r$ and $r_{i}$ as "unit matrix", i.e., for any 
$x \in J \cup \mathbb{N}$ and $y \in Y^{\prime}$ let $r(x, y)=r_{i}(x, y)=1$ if $y=\pi(x)$, and $r(x, y)=r_{i}(x, y)=0$ otherwise. In the picture, $r_{i}$ is denoted as $\mathbb{I}_{\pi}$.

Further, for all $b \in B$ and $x \in \mathbb{N}$ let $r_{i}(b, x)=v(b)$. On $\mathbb{N} \times \mathbb{N}$, let $r_{i}$ be the same relation as used in the proof of Theorem 3.5. Recall that $\left(\mathbb{N}, r_{i}, \mathbb{N}\right)$ is biextensional. Finally, put $r_{i} \equiv 0$ on $J \times \mathbb{N}$.

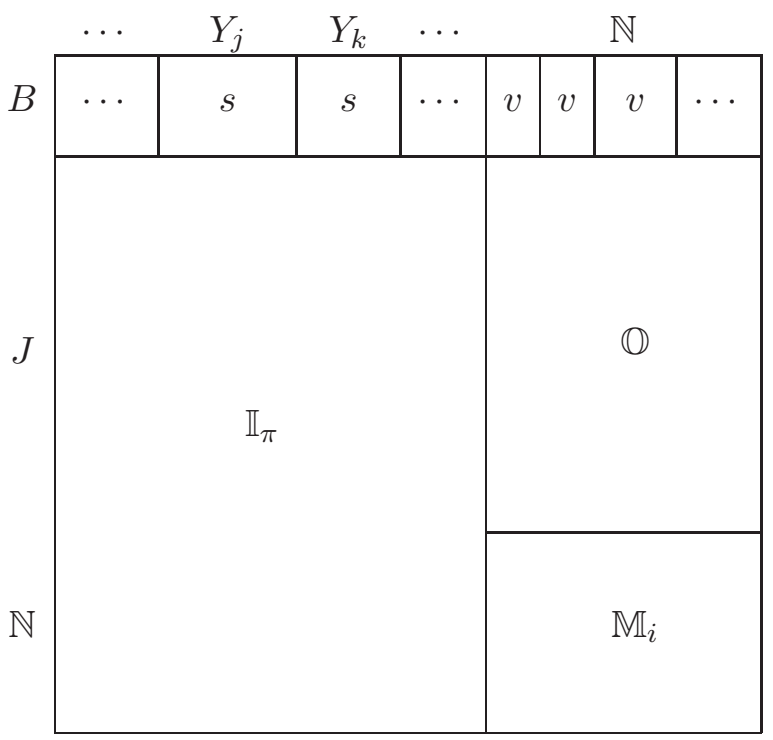

Observe that for each $b \in B$ either $s(b,-)$ is the constant-0 function, which implies that $r_{i}(b,-)=r(b,-)$ is constantly 0 on $Y^{\prime}$, or else $r_{i}(b, y)=r(b, y)=1$ for infinitely many $y \in Y^{\prime}$. It follows that $\mathrm{C}_{i}$ and $\mathrm{C}$ are biextensional.

Now define morphisms $\varphi_{i}: \mathrm{C}_{i} \rightarrow \mathrm{C}_{i+1}$ and $\psi_{i}: \mathrm{C}_{i} \rightarrow \mathrm{C}$ such that $\varphi_{i}^{+}=\psi_{i}^{+}=i d_{B \cup J \cup \mathbb{N}}$, $\varphi_{i}^{-}$and $\psi_{i}^{-}$are the identity on $Y^{\prime}$, and $\varphi_{i}^{-}(x)=x+1$ for each $x \in \mathbb{N}$. Then $\left(\psi_{i}: \mathrm{C}_{i} \rightarrow \mathrm{C}\right)_{i \geq 1}$ is the colimit of the sequence $\left(\mathrm{C}_{i}, \varphi_{i}\right)_{i \geq 1}$. Next we define a morphism $\psi: \mathrm{F} \rightarrow \mathrm{C}$ by letting $\psi^{+}=i d_{B}$ and $\psi^{-}(y, j)=y$ for each $y \in Y, j \in J$. Since $\mathbf{F}$ is finite, there is a morphism from $\mathrm{F}$ into some $C_{i}$, which implies a contradiction by the choice of $v$.

Secondly, suppose that $B$ is infinite. For a subset $S \subseteq B$, we call two elements $y, y^{\prime} \in Y$ $S$-equivalent if $r(-, y)$ and $r\left(-, y^{\prime}\right)$ coincide on $S$. Now split $B=A^{\prime} \cup\left\{a_{i} \mid i \in \mathbb{N}\right\}$ with $A^{\prime} \cap\left\{a_{i} \mid i \in \mathbb{N}\right\}=\emptyset$. For each $i \geq 1$, let $A_{i}=A^{\prime} \cup\left\{a_{j} \mid 1 \leq j \leq i\right\}$, and let $X_{i}$ contain from each $A_{i}$-equivalence class in $Y$ exactly one element.

Let $r_{i}$ be $r$ restricted to $A_{i} \times X_{i}$, and put $\mathrm{C}_{i}=\left(A_{i}, r_{i}, X_{i}\right)$. Then $\mathrm{C}_{i}$ is separable, since $B$ is separable and $X_{i}$ intersects each $A_{i}$-equivalence class, and $C_{i}$ is extensional since $X_{i}$ contains from each $A_{i}$-equivalence class at most one element.

Now let $\varphi_{i}^{+}$be the identity, and for $x_{i+1} \in X_{i+1}$ let $\varphi_{i}^{-}\left(x_{i+1}\right)=x_{i}$ if $x_{i} \in X_{i}$ lies in the $A_{i}$-equivalence class of $x_{i+1}$. The sequence $\left(\mathrm{C}_{i}, \varphi_{i}\right)_{i \geq 1}$ has a colimit $\mathrm{C}=(A, r, X)$ in $\mathbf{i E}$, and clearly $\mathrm{C}$ is separable. Also, we may assume that $\mathrm{C}$ is obtained by Construction 3.4; then $A=B$.

There is a unique Chu morphism $\varphi$ from $\mathrm{F}$ to $\mathrm{C}$ with $\varphi^{+}$the identity on $B$; here the existence of $\varphi^{-}$follows from $F$ being discrete and the uniqueness from $F$ being extensional. Hence there are an $i \geq 1$ and $\varphi: \mathrm{F} \rightarrow \mathrm{C}_{i}$ which make the diagram commute, and this implies a contradiction about $a_{i+1}$ as in the proof of Theorem 4.1 . 


\section{Bifinite Chu spaces}

In this section, we will investigate Chu spaces which are, intuitively and in a categorytheoretic sense, countable objects and approximable by the finite objects in the category. That is, we will define bifinite Chu spaces as colimits of a sequence of (strongly) finite Chu spaces. We will then show that this subcategory of $\mathbf{i C}$ contains a universal homogeneous object.

Recall that the finite objects of $\mathbf{i C}$ may have an infinite attribute set, if $\Sigma$ is infinite (cf. Example 4.3). For technical reasons (cf. the proofs of Theorem 5.3 and Proposition 5.5), we will need that the objects employed here have a finite and non-empty set of attributes. We will call a space $\mathrm{F}$ in $\mathbf{i C}$ strongly finite, if $\mathbf{F}$ is a finite object in $\mathbf{i C}$ and a finite $\mathrm{Chu}$ space with non-empty set of attributes. Clearly, if $\Sigma$ is finite, the finite and the strongly finite objects of $\mathbf{i} \mathbf{C}$ with non-empty sets of attributes coincide.

Definition 5.1. A Chu space in $\mathbf{i C}$ is called bifinite if it is isomorphic to the colimit (with respect to $\mathbf{i E}$ ) of a chain of stronly finite objects in $\mathbf{i C}$. The corresponding full subcategory of bifinite $\mathrm{Chu}$ spaces of $\mathbf{C}$ and $\mathbf{i} \mathbf{C}$ are denoted as $\mathbf{C}_{\mathrm{bif}}$ and $\mathbf{i} \mathbf{C}_{\mathrm{bif}}$, respectively.

As an example, consider the sequence of strongly finite biextensional spaces $\left(\mathrm{C}_{i}, \varphi_{i}\right)_{i \geq 1}$ described in the proof of Theorem 3.1. As shown there, this sequence has no colimit in the category iC. But by Theorem 3.4, the sequence has a colimit with respect to the category iE. This colimit thus belongs to $\mathbf{i C}_{\text {bif }}$. Moreover, we will see below in Theorem 5.3 , that this space is also a colimit of the given sequence with respect to the category $\mathbf{i} \mathbf{C}_{\mathrm{bif}}$.

Recall that any finite object of $\mathbf{i C}$ is extensional, hence any bifinite Chu space is also extensional. It would not be interesting to formulate the concept of bifinite spaces in $\mathbf{i E}$ or $\mathbf{i B}$, i.e. as colimits of chains of finite objects of $\mathbf{i E}$ resp. $\mathbf{i B}$ : By Theorems 4.5 and 4.7 , these finite objects are discrete. One can show that colimits of chains of discrete extensional objects are again discrete and extensional. Hence any two such 'bifinite' objects (in $\mathbf{i E}$ or iB) with countably infinite object set are isomorphic by Remark 4.6. In contrast, we show that $\mathbf{i} \mathbf{C}_{\text {bif }}$ is very large:

Proposition 5.1. $\mathbf{i C}_{\mathrm{bif}}$ contains at least continuum many non-isomorphic objects.

Proof. Consider a strictly increasing sequence of finite subsets $A_{1} \subset A_{2} \subset \ldots \subset \mathbb{N}$ of $\mathbb{N}$. We define a sequence $\left(\mathrm{C}_{i}, \varphi_{i}\right)_{i \geq 1}$ as follows. For each $i \geq 1$, let $\mathrm{C}_{i}=\left(A_{i}, r_{i}, X_{i}\right)$ with $X_{i}=\{1, \ldots, i\}$ and $r_{i}(a, j)=1$ if $a \in A_{j}$ and $r_{i}(a, j)=0$ otherwise, for any $a \in A_{i}, j \in X_{i}$. We let $\varphi_{i}^{+}$be the inclusion mapping, $\varphi_{i}^{-}(j)=j$ if $1 \leq j \leq i$, and $\varphi_{i}^{-}(i+1)=i$. As colimit of this sequence of strongly finite objects we obtain, up to isomorphism, $\left(\mathrm{C}_{i} \stackrel{\psi_{i}}{\longrightarrow} \mathrm{C}\right)_{i \geq 1}$ with $\mathrm{C}=(\mathbb{N}, r, \mathbb{N} \cup\{\infty\}), r(a, j)=1$ if $a \in A_{j}$ and $r(a, j)=0$ otherwise, for any $a, j \in \mathbb{N}$, further $r(-, \infty)=1$, and $\psi_{i}^{+}$inclusion, $\psi_{i}^{-}(j)=j$ if $1 \leq j \leq i$ and $\psi_{i}^{-}(j)=i$ if $i<j \in \mathbb{N} \cup\{\infty\}$. Note that in $\mathrm{C}$ the set $\{a \in \mathbb{N} \mid r(a, i)=1\}$ equals $A_{i}$ if $i \in \mathbb{N}$, and $\mathbb{N}$ if $i=\infty$. Hence the bifinite space $\mathbf{C}$ constructed in this way determines the sequence of subsets $\left(A_{i}\right)_{i \geq 1}$ uniquely, and two different sequences give rise to non-isomorphic bifinite spaces. Since there are continuum many such sequences, the result follows.

Remark 5.2. By cardinality arguments, one can show that up to isomorphism $\mathbf{i} \mathbf{C}_{\text {bif }}$ has size $|\Sigma|^{\omega}$; this equals the continuum if $\Sigma$ has size at most continuum.

With the restriction of objects to bifinite Chu spaces, colimits now exist, in contrast to Theorem 3.1 , 
Theorem 5.3. Colimits exist in $\mathbf{i C}_{\mathrm{bif}}$.

The technical content of the result is that $\mathbf{i} \mathbf{C}_{\mathrm{bif}}$ is closed in $\mathbf{i C}$ and in $\mathbf{i E}$ with respect to taking colimits of sequences in $\mathbf{i} \mathbf{C}_{\mathrm{bif}}$, and these colimits taken in $\mathbf{i} \mathbf{E}$ constitute the colimits of the given sequences with respect to $\mathbf{i C}_{\mathrm{bif}}$.

Proof. First, let $\left(\mathrm{C}_{i}, \varphi_{i}\right)_{i \geq 1}$ be an $\omega$-sequence in $\mathbf{i C}$ with finite objects $\mathrm{C}_{i}=\left(A_{i}, r_{i}, X_{i}\right)$, $X_{i} \neq \emptyset$, and each $\varphi_{i}^{+}$being an inclusion. By Theorem 4.1 each $C_{i}$ is extensional. Define $\mathrm{C}=(A, r, X)$ and $\psi_{i}: \mathrm{C}_{i} \rightarrow \mathrm{C}(i \geq 1)$ as in Construction 3.2. By Theorem 3.4, the cocone $\left(\mathrm{C}_{i} \stackrel{\psi_{i}}{\longrightarrow} \mathrm{C}\right)_{i \geq 1}$ is a colimit of $\left(\mathrm{C}_{\mathbf{i}}, \varphi_{i}\right)_{i \geq 1}$ in $\mathbf{i E}$. We claim that it is also a colimit of this sequence in $\mathbf{i C}_{\text {bif }}$.

First we show that each $\psi_{i}: \mathrm{C}_{i} \rightarrow \mathrm{C}$ is a morphism in $\mathbf{i C}$, i.e., that $\psi_{i}^{-}: X \rightarrow X_{i}$ is onto. Choose any $x_{i} \in \mathrm{C}_{i}$. Clearly, since all $\varphi_{j}^{-}: X_{j+1} \rightarrow X_{j}(j \geq 1)$ are onto, there is a sequence $\left(x_{j}\right)_{j \geq 1}$ with $x_{j} \in X_{j}$ and $\varphi_{j}^{-}\left(x_{j+1}\right)=x_{j}$ for each $j \geq 1$. Then $\tilde{x}=\left(x_{j}\right)_{j \geq 1} \in X$ and $\psi_{i}^{-}(\tilde{x})=x_{i}$, as needed.

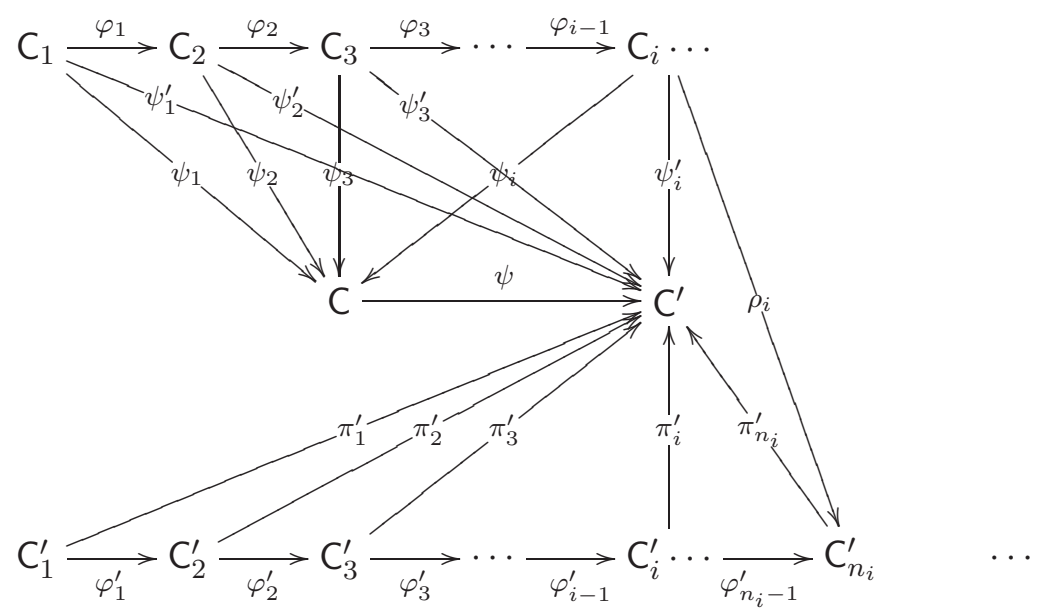

For universality, let $\mathrm{C}^{\prime}=\left(A^{\prime}, r^{\prime}, X^{\prime}\right) \in \mathbf{i} \mathbf{C}_{\text {bif }}$ and $\left(\mathrm{C}_{i} \stackrel{\psi_{i}^{\prime}}{\longrightarrow} \mathrm{C}^{\prime}\right)_{i \geq 1}$ be a cocone. Define $\psi: \mathbf{C} \rightarrow \mathrm{C}^{\prime}$ as in the proof of Theorem 3.4. Then $\psi$ is a mediating morphism in $\mathbf{i E \text { , and }}$ it only remains to show surjectivity of $\psi^{-}: X^{\prime} \rightarrow X$. Choose any $\tilde{x}=\left(x_{j}\right)_{j \geq 1} \in X$. Since $\mathrm{C}^{\prime} \in \underset{\mathbf{i}^{\prime}}{\mathbf{\mathbf { C } _ { \mathrm { bif } }}}$, we can choose a chain of finite objects $\mathrm{C}_{i}^{\prime}=\left(A_{i}^{\prime}, r_{i}^{\prime}, X_{i}^{\prime}\right)$ and monics $\varphi_{i}^{\prime}$ such that $\left(\mathrm{C}_{i}^{\prime} \stackrel{\pi^{\prime} i}{\longrightarrow} \mathrm{C}^{\prime}\right)_{i \geq 1}$ is a colimit of this chain in iC. Again we can assume that the $\varphi_{i}^{\prime+} \mathrm{S}$ are inclusions and that the colimit $\left(\mathrm{C}_{i}^{\prime} \stackrel{\pi^{\prime} i}{\longrightarrow} \mathrm{C}^{\prime}\right)_{i \geq 1}$ is given as in Construction 3.2. Each $\mathrm{C}_{i}$ is a finite object. Observing the morphisms $\psi_{i}^{\prime}: \mathrm{C}_{i} \rightarrow \mathrm{C}^{\prime}=\operatorname{colim}_{i}\left(\mathrm{C}_{i}^{\prime}, \varphi_{i}^{\prime}\right)$, we obtain a sequence of numbers $n_{1}<n_{2}<\ldots$ and monics $\rho_{i}: \mathrm{C}_{i} \rightarrow \mathrm{C}_{n_{i}}^{\prime}$ which make the diagram commute, that is, for each $i \geq 1$ we have $\psi_{i}^{\prime}=\pi_{n_{i}}^{\prime} \circ \rho_{i}$. For $i<j$ let $\varphi_{i, j}^{\prime}$ be the composition of $\varphi_{i}^{\prime}$ up to $\varphi_{j-1}^{\prime}$ from $\mathrm{C}_{i}^{\prime}$ to $\mathrm{C}_{j}^{\prime}$. Since the diagram commutes and the $\pi_{i}^{\prime}$ are monic, we obtain

$$
\varphi_{n_{i}, n_{i+1}}^{\prime} \circ \rho_{i}=\rho_{i+1} \circ \varphi_{i} \text {, for each } i \geq 1 \text {. }
$$

Now consider the collection $K$ of all finite sequences $\left(x_{1}^{\prime}, \ldots, x_{m}^{\prime}\right)$ such that

$$
x_{j}{ }^{\prime} \in X_{n_{j}}^{\prime} \text { and } \rho_{j}^{-}\left(x_{j}{ }^{\prime}\right)=x_{j} \text { for each } j \leq m
$$

and

$$
\varphi_{n_{j}, n_{j+1}}^{\prime}{ }^{-}\left(x_{j+1}^{\prime}\right)=x_{j}^{\prime} \text { for each } j<m
$$


We claim that there are arbitrarily long sequences. Choose any $m \in \mathbb{N}$. Since $\rho_{j}^{-}$is surjective, there is $x_{m}^{\prime} \in X_{n_{m}}^{\prime}$ satisfying $\rho_{m}^{-}\left(x_{m}^{\prime}\right)=x_{m}$. Now put $x_{j}^{\prime}=\varphi_{n_{j}, n_{m}}^{\prime}{ }^{-}\left(x_{m}^{\prime}\right)$ for each $1 \leq j<m$. By $(*)$, inductively we obtain

$$
\rho_{j}^{-}\left(x_{j}^{\prime}\right)=\rho_{j}^{-} \circ \varphi_{n_{j}, n_{j+1}}^{\prime}{ }^{-}\left(x_{j+1}^{\prime}\right)=\varphi_{j}^{-} \circ \rho_{j+1}^{-}\left(x_{j+1}^{\prime}\right)=\varphi_{j}^{-}\left(x_{j+1}\right)=x_{j},
$$

and our requirements $(* *)$ follow.

Now consider $K$ with the extension order. The sets $X_{j}^{\prime}$ are finite since the $C_{j}^{\prime}$ are strongly finite objects. So, $K$ is an infinite finite-branching tree. By König's lemma, there is an infinite branch in this tree. Hence there is an infinite sequence $\left(x_{j}^{\prime}\right)$ satisfying requirements $(* *)$ for each $j \geq 1$. Now fill up this sequence with the necessary additional elements from $X_{i}^{\prime}$ to obtain an element $\tilde{x^{\prime}}=\left(x_{j}^{\prime \prime}\right)_{j \geq 1} \in X^{\prime}$ satisfying $x_{n_{j}}^{\prime \prime}=x_{j}^{\prime}$ for each $j \geq 1$. We claim that $\psi^{-}\left(\tilde{x^{\prime}}\right)=\tilde{x}$. Indeed, choose any $i \geq 1$. Then $\psi_{i}^{\prime-}\left(\tilde{x^{\prime}}\right)=\rho_{i}^{-} \circ \pi_{n_{i}}^{\prime}{ }^{-}\left(\tilde{x^{\prime}}\right)=\rho_{i}^{-}\left(x_{n_{i}}^{\prime \prime}\right)=$ $\rho_{i}^{-}\left(x_{i}^{\prime}\right)=x_{i}$. This proves our claim.

Secondly, consider an arbitrary $\omega$-sequence $\left(\mathrm{C}_{i}, \varphi_{i}\right)_{i \geq 1}$ in $\mathbf{i} \mathbf{C}_{\text {bif }}$, and let $\left(\mathrm{C}_{i} \stackrel{\psi_{i}}{\longrightarrow} \mathrm{C}\right)_{i \geq 1}$ be its colimit in $\mathbf{i E}$. We claim that this is also the colimit in $\mathbf{i} \mathbf{C}_{\text {bif }}$. As before, we can show that each $\psi_{i}: \mathbf{C}_{i} \rightarrow \mathbf{C}$ is a morphism in $\mathbf{i C}$. Now we continue in a standard way.

For each $i \geq 1$, since $\mathrm{C}_{i} \in \mathbf{i} \mathbf{C}_{\text {bif }}$, we can write $\mathrm{C}_{i}$ as a colimit of a sequence of strongly finite objects $\left(\mathrm{C}_{i j}\right)_{j \geq 1}$ in $\mathbf{i C}$ and in $\mathbf{i E}$. By a diagonal argument, there is a sequence of numbers $n_{1}<n_{2}<\ldots$ such that the spaces $\left(C_{i, n_{i}}\right)_{i \geq 1}$ form a sequence in $\mathbf{i C}$ whose colimit, if it exists, is also colimit of the sequence $\left(C_{i}, \varphi_{i}\right)_{i \geq 1}$ in $\mathbf{i C}$, and whose colimit in $\mathbf{i E}$ is $\mathbf{C}$. Hence $\mathbf{C} \in \mathbf{i} \mathbf{C}_{\text {bif }}$, and by our first part, $\mathbf{C}$ is the colimit of the sequence $\left(\mathrm{C}_{i, n_{i}}\right)_{i \geq 1}$ also in $\mathbf{i C}_{\mathrm{bif}}$. Thus $\mathbf{C}$ is the colimit of $\left(\mathbf{C}_{i}, \varphi_{i}\right)_{i \geq 1}$ in $\mathbf{i} \mathbf{C}_{\mathrm{bif}}$.

To make the paper self-contained, we recall briefly a result of Droste and Göbel [6] concerning the existence of a universal, homogeneous object in an algebroidal category. Let $\mathbf{G}$ be a category in which all the morphisms are monic, and $\mathbf{G}^{*}$ a full subcategory of $\mathbf{G}$. Individually, an object $U$ of $\mathbf{G}$ is called

- $\mathbf{G}^{*}$-universal if for any object $A$ in $\mathbf{G}^{*}$, there is a morphism $f: A \rightarrow U$;

- $\mathbf{G}^{*}$-homogeneous if for any $A$ in $\mathbf{G}^{*}$ and any pair $f, g: A \rightarrow U$, there is an isomorphism $h: U \rightarrow U$ such that $f=h \circ g$;

Intuitively, $\mathbf{G}^{*}$-homogeneity means that each isomorphism between two $\mathbf{G}^{*}$-substructures of $U$ extends to an automorphism of $U$; this means that $U$ has maximal possible degree of symmetry.

Collectively, the category $\mathbf{G}^{*}$ is said to have the amalgamation property if for any $f_{1}: A \rightarrow B_{1}, f_{2}: A \rightarrow B_{2}$ in $\mathbf{G}^{*}$, there exist $g_{1}: B_{1} \rightarrow B, g_{2}: B_{2} \rightarrow B$ in $\mathbf{G}^{*}$ such that $g_{1} \circ f_{1}=g_{2} \circ f_{2}$.

Definition 5.2. Let $\mathbf{G}$ be a category in which all morphisms are monic. Then $\mathbf{G}$ is called algebroidal, if $\mathbf{G}$ has the following properties:

(1) $\mathbf{G}$ has a weakly initial object,

(2) Every object of $\mathbf{G}$ is a colimit of an $\omega$-chain of finite objects,

(3) Every $\omega$-sequence of finite objects has a colimit, and

(4) The number of finite objects of $\mathbf{G}$, up to isomorphism, is countable and between any pair of finite objects there exist only countably many morphisms.

Theorem 5.4. (Droste and Göbel) Let $\mathbf{G}$ be an algebroidal category with all morphisms monic. Let $\mathbf{G}_{f}$ be the full subcategory of finite objects of $\mathbf{G}$. Then there exists a $\mathbf{G}$-universal, 
$\mathbf{G}_{f}$-homogeneous object iff $\mathbf{G}_{f}$ has the amalgamation property. Moreover, in this case the $\mathbf{G}$-universal, $\mathbf{G}_{f}$-homogeneous object is unique up to isomorphism.

Proposition 5.5. The category $\mathbf{i C}_{\text {bif }}$ contains an initial object. The strongly finite objects of $\mathbf{i C}$ are precisely the finite objects of $\mathbf{i C}_{\text {bif }}$. If $\Sigma$ is countable, there are only countably many non-isomorphic finite objects in $\mathbf{i} \mathbf{C}_{\mathrm{bif}}$. Between any pair of finite objects there are only finitely many injections. Moreover, the finite objects of $\mathbf{i C}_{\mathrm{bif}}$ have the amalgamation property.

Proof. The space $(\emptyset, \emptyset,\{x\})$ is the initial object of $\mathbf{i} \mathbf{C}_{\mathrm{bif}}$, since all spaces in $\mathbf{i} \mathbf{C}_{\mathrm{bif}}$ have nonempty attribute sets. Next, we show only the amalgamation property; the rest is easy to see. Suppose $\mathrm{C}:=(A, r, X), \mathrm{C}_{1}:=\left(A_{1}, r_{1}, X_{1}\right)$, and $\mathrm{C}_{2}:=\left(A_{2}, r_{2}, X_{2}\right)$ are strongly finite objects in iC such that $A=A_{1} \cap A_{2}$, and let $\varphi_{1}: \mathrm{C} \rightarrow \mathrm{C}_{1}$ and $\varphi_{2}: \mathrm{C} \rightarrow \mathrm{C}_{2}$ be morphisms in $\mathbf{i C}$.

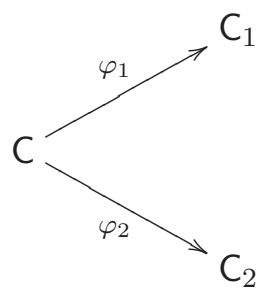

Construct $\mathrm{C}^{\prime}:=\left(A^{\prime}, r^{\prime}, X^{\prime}\right)$ as:

$$
\begin{aligned}
A^{\prime} & =A_{1} \cup A_{2} \\
X^{\prime} & =\left\{\left(x_{1}, x_{2}\right) \in X_{1} \times X_{2} \mid \varphi_{1}^{-}\left(x_{1}\right)=\varphi_{2}^{-}\left(x_{2}\right)\right\} \\
r^{\prime}\left(a,\left(x_{1}, x_{2}\right)\right) & =r_{1}\left(a, x_{1}\right) \text { if } a \in A_{1} \\
r^{\prime}\left(a,\left(x_{1}, x_{2}\right)\right) & =r_{2}\left(a, x_{2}\right) \text { if } a \in A_{2} .
\end{aligned}
$$

Note that in case $a \in A_{1} \cap A_{2}$, we have

$$
\begin{aligned}
r_{1}\left(a, x_{1}\right) & =r\left(a, \varphi_{1}^{-}\left(x_{1}\right)\right) \\
& =r\left(a, \varphi_{2}^{-}\left(x_{2}\right)\right) \\
& =r_{2}\left(\varphi_{2}^{+}(a), x_{2}\right) \\
& =r_{2}\left(a, x_{2}\right) .
\end{aligned}
$$

To see that $\mathrm{C}^{\prime}$ is extensional, suppose $\left(x_{1}, x_{2}\right),\left(y_{1}, y_{2}\right) \in X^{\prime}$ are such that $r^{\prime}\left(a,\left(x_{1}, x_{2}\right)\right)=$ $r^{\prime}\left(a,\left(y_{1}, y_{2}\right)\right)$ for all $a \in A_{1} \cup A_{2}$. By the definition of $C^{\prime}$, then, for each $a \in A_{1}$, we have $r_{1}\left(a, x_{1}\right)=r_{1}\left(a, y_{1}\right)$. By the extensionality of $\mathrm{C}_{1}$, we have $x_{1}=y_{1}$. Similarly, by the extensionality of $\mathrm{C}_{2}$, we have $x_{2}=y_{2}$ and so $\left(x_{1}, x_{2}\right)=\left(y_{1}, y_{2}\right)$, as required.

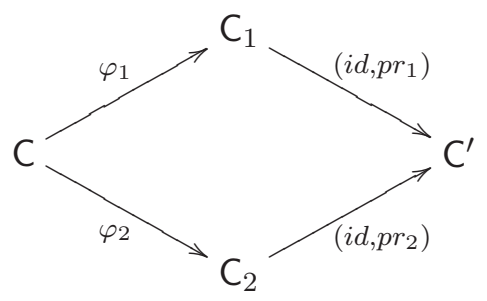

It is easy to check further that $\left(i d, p r_{1}\right): \mathrm{C}_{1} \rightarrow \mathrm{C}^{\prime}$ and $\left(i d, p r_{2}\right): \mathrm{C}_{2} \rightarrow \mathrm{C}^{\prime}$ are morphisms in iC. 
By Proposition 5.5, the following result immediately follows from Theorem 5.4,

Theorem 5.6. Let $\Sigma$ be countable. Then $\mathbf{i C}_{\mathrm{bif}}$ is an algebroidal category containing a universal homogeneous object $U$. Moreover, $U$ is unique up to isomorphism.

Since $\mathbf{i} \mathbf{C}_{\text {bif }}$ contains spaces with an attribute set of size continuum, it follows that the attribute set of $U$ also has size continuum. However, we just note that since the proof of Theorem 5.4 is constructive, we can construct a sequence $\left(C_{i}, \varphi_{i}\right)_{i>1}$ whose colimit is the universal homogeneous object $U$.

We remark that $\mathbf{i} \mathbf{C}_{\text {bif }}$ does not contain all countable extensional Chu spaces (just as not all countable cpos are SFP). Let $\mathrm{C}=(\mathbb{N}, r, \mathbb{N})$ be the biextensional Chu space described in the proof of Theorem 3.1. We claim that $\mathrm{C}$ is not bifinite.

Indeed, choose the sequence $\left(C_{i}, \varphi_{i}\right)_{i>1}$, the space $C^{\prime}=\left(\mathbb{N}, r^{\prime}, \mathbb{N} \cup\{t\}\right)$ and the monics $\psi_{i}^{\prime}: \mathrm{C}_{i} \rightarrow \mathrm{C}^{\prime}$ as in the proof of Theorem 3.1. By Theorem 3.4, $\left(\psi_{i}^{\prime}: \mathrm{C}_{i} \rightarrow \mathrm{C}^{\prime}\right)_{i \geq 1}$ is the colimit of the chain $\left(\mathrm{C}_{i}, \varphi_{i}\right)_{i \geq 1}$ in $\mathbf{i E}$. By Theorem 5.3, this is also the colimit of the sequence of finite spaces $\mathbf{C}_{i}$ in the category $\mathbf{i} \mathbf{C}_{\text {bif }}$. Consider the morphisms $\left(\psi_{i}: \mathbf{C}_{i} \rightarrow \mathbf{C}\right)_{i \geq 1}$ described in the proof of Theorem 3.1. Now if $\mathrm{C}$ was bifinite, there would be a unique morphism $\psi: \mathbf{C}^{\prime} \rightarrow \mathbf{C}$ in $\mathbf{i C}_{\text {bif }}$ making the diagram commute. But then $\psi^{+}=i d_{\mathbb{N}}$, and $\psi^{-}(n)=t$ for some $n \in \mathbb{N}$, yielding $0=r(n+1, n)=r^{\prime}(n+1, t)=1$, a contradiction.

\section{Concluding Remarks}

One specific motivation for considering the notion of bifinite Chu space is for modeling linear logic [12, for which tensor and linear negation should also be brought into the picture. Although the category of bifinite Chu spaces is monoidal, it is not monoidal closed [11. Also, the construction of linear negation cannot be accounted for nicely in bifinite Chu spaces either. In spite of these, one has to look at the bigger picture and consider our results in the context of Chu spaces as a general framework for studying the dualities of objects and properties, points and open sets, and terms and types, under rich mathematical contexts. This view has already been made amply clear by Pratt $18,19,20,21,22,23,24$. Traditionally, the study on Chu spaces had a non-constructive flavor. This paper provides (1) a basis for a more constructive analysis of categories of Chu spaces collectively; (2) a framework in which finite Chu spaces can be used to approximate infinite ones as colimits of $\omega$-chains of finite Chu spaces; (3) the "completeness" of the delineated categories under the colimit construction. On the technical side, the development of our framework hinges upon the adoption of monic morphisms as the basic steps for approximation at the structural level. It is certainly reasonable to consider other possible notions of "approximation" as well, such as "regular mono" or more generally "embedding-projection pair", of which monic morphism can be regarded as a special case.

Acknowledgment. The authors would like to thank F. Lamarche and the anonymous referees for valuable feedback.

\section{REFERENCES}

[1] R. Amadio and P.-L. Curien, Domains and Lambda-Calculi. Cambridge University Press, 1998.

[2] M. Barr. *-Autonomous categories, with an appendix by Po Hsiang Chu. Lecture Notes in Mathematics, Vol. 752, Springer-Verlag, 1979. 
[3] M. Barr. *-Autonomous categories and linear logic. Mathematical Structures in Computer Science, Vol. 1, pp. 159-178, 1991.

[4] H. Devarajan, D. Hughes, G. Plotkin, V. Pratt. Full completeness of the multiplicative linear logic of Chu spaces. 14th Symposium on Logic in Computer Science (Trento, 1999), 234-243, IEEE Computer Soc., Los Alamitos, CA, 1999.

[5] M. Erné. General Stone duality. Topology and Its Applications, Vol. 137, pp. 125-158, 2004.

[6] M. Droste and R. Göbel. Universal domains and the amalgamation property. Mathematical Structures in Computer Science. 3:137-159, 1993.

[7] M. Droste. Universal homogeneous causal sets. Journal of Mathematical Physics, 46:1-10, 2005.

[8] B. Ganter and R. Wille. Formal Concept Analysis. Springer-Verlag, 1999.

[9] P. Hitzler and G.-Q. Zhang. A cartesian closed category of approximable concept structures. In Pfeiffer and Wolff (eds.), Proceedings of the International Conference on Conceptual Structures, Huntsville, Alabama, USA, July 2004. Lecture Notes in Artificial Intelligence, Vol. 3127, pages 170-185, 2004.

[10] P. Hitzler, M. Krötzsch and G.-Q. Zhang. A categorical view on algebraic lattices in Formal Concept Analysis. Fundamenta Informaticae, Volume 74 (2-3), pp. 301 - 328, 2006.

[11] F. Huang, M. Droste and G.-Q. Zhang. A monoidal category of bifinite Chu spaces. Electronic Notes in Theoretical Computer Science, Vol. 212, pp. 285-297, 2008.

[12] F. Lamarche. Dialectics: a model of linear logic and PCF. Submitted to MSCS.

[13] F. Lamarche. From Chu spaces to cpos. Theory and Formal Methods of Computing 94, Imperial College Press, pp. 283-305, 1994.

[14] S. Mac Lane. Categories for the Working Mathematician. Springer-Verlag, 1971.

[15] G. Plotkin. A powerdomain construction. SIAM J. Comput., Vol. 5, pp. 452-487, 1976.

[16] G. Plotkin. $T^{\omega}$ as a universal domain. J. Comp. Sys. Sci. Vol. 17, pp. 209-236, 1978.

[17] G. Plotkin. Notes on the Chu construction and recursion. http://boole.stanford.edu/pub/gdp.pdf (accessed Jan 2007.)

[18] V. Pratt. Chu spaces. School on Category Theory and Applications, Textos Mat. SCr. B, 21, 39-100, Univ. Coimbra, Coimbra, 1999.

[19] V. Pratt. Higher dimensional automata revisited. Math. Structures Comput. Sci. Vol. 10, pp. 525-548, 2000 .

[20] V. Pratt. Chu spaces from the representational viewpoint. Ann. Pure Appl. Logic, Vol. 96, pp. 319-333, 1999.

[21] V. Pratt. Towards full completeness of the linear logic of Chu spaces. Mathematical Foundations of Programming Semantics (Pittsburgh, PA, 1997), Electronic Notes in Theoretical Computer Science, Vol. 7, 18 pp., 1997.

[22] V. Pratt. The Stone gamut: a coordinatization of mathematics. Proceedings of 10th Annual Symposium on Logic in Computer Science, pp. 444-454, 1995.

[23] V. Pratt. Chu spaces and their interpretation as concurrent objects. Lecture Notes in Comput. Sci. Vol. 1000, pp. 392-405, 1995.

[24] V. Pratt. Chu spaces as a semantic bridge between linear logic and mathematics. Theoretical Computer Science, Vol. 294, pp. 439-471, 2003.

[25] D. Scott. Data types as lattices. SIAM J. Comput., Vol. 5, pp. 522-586, 1976.

[26] D. Scott. Domains for denotational semantics. Automata, Languages and Programming (Aarhus, 1982), pp. 577-613, Lecture Notes in Comput. Sci., 140, Springer, Berlin-New York, 1982.

[27] G.-Q. Zhang. Chu spaces, concept lattices, and domains. In: Proceedings of the 19th Conference on the Mathematical Foundations of Programming Semantics, March 2003, Montreal, Canada. Electronic Notes in Theoretical Computer Science Vol. 83, 2004, 17 pages.

[28] G.-Q. Zhang and G. Shen. Approximable concepts, Chu spaces, and information systems. In V. De Paiva and V. Pratt (edts.) Theory and Applications of Categories, Special Volume on Chu Spaces: Theory and Applications, Vol. 17, No. 5, pp. 80-102, 2006.

This work is licensed under the Creative Commons Attribution-NoDerivs License. To view a copy of this license, visit http://creativecommons.org/licenses/by-nd/2.0/ or send a letter to Creative Commons, 171 Second St, Suite 300, San Francisco, CA 94105, USA, or Eisenacher Strasse 2, 10777 Berlin, Germany 